\title{
NOVEL APPROACH TO EXTRACT DENSE FULL-FIELD DYNAMIC PARAMETERS OF LARGE-SCALE BRIDGES USING SPATIAL SEQUENCE VIDEO
}

\author{
Guojun DENG(101,2,3, Zhixiang ZHOU(1) 1,3*, \\ Shuai SHAO ${ }^{1,3}$, Xi CHU (D) 3 , Peng DU (D) 3 \\ ${ }^{1}$ College of Civil and Transportation Engineering, Shenzhen University, Shenzhen, China \\ ${ }^{2}$ China Merchants Chongqing Communications Technology Research \& Design Institute Co., Ltd., \\ Chongqing, China \\ ${ }^{3}$ School of Civil Engineering, Chongqing Jiaotong University, Chongqing, China
}

Received 15 June 2020; accepted 2 August 2021

\begin{abstract}
This study proposes the use of a high-speed camera as a holographic visual sensor to obtain the dense full-field dynamic parameters of the main beam of a bridge by the field of view through uniaxial rotation photography. Based on the basic principle that the frequency and mode of a structure are inherent characteristics, the mode coordinates obtained from each field of view are unified, normalized, and matched according to the same name pixels to obtain the dense fullfield dynamic parameters of the entire bridge. The frequency and first three order modes of a self-anchored suspension test bridge are collected by the method proposed in this study. The frequency comparison between the accelerometers and dial gauges is within 3\%, and the mode shapes are more holographic and more realistic than those obtained by limited measuring points. In addition, the difference in the curvature mode under various damage conditions obtained by limited measurement points is compared with that obtained by the method proposed in this study. Results shows that the dense full-field modal curvature difference can reflect the change in the damage location even in a low order, which means the sensitivity of the change of damage location in low-order modal.
\end{abstract}

Keywords: structural health monitoring, holographic visual sensor, uniaxial rotation photography, structural damage identification.

\section{Introduction}

With engineers' and researchers' deepening understanding of civil structures, vibration analysis occupies an increasingly important position in the design research of civil construction, operation, and maintenance. Modal analysis is a method of analyzing structural dynamics, using the inherent characteristics of a structure produced recently, including dynamic property natural frequencies, damping ratio, and mode shapes, to describe the structure. The main purpose of modal analysis is to obtain the modal parameters of a system structure to describe its dynamic characteristics.

Vibration testing methods can be divided into two types, that is, contact measurement methods and noncontact measurement methods. Contact measurement methods rely mainly on sensors and strain gauges to collect vibration data, which must be set on the surface of a structure. Occasionally, an entire structure surface must be covered with such instruments to obtain complete data, which is labor intensive. In flexible or lightweight structures, the introduction of data transmission lines and load quality can seriously affect measurement results. Meanwhile, noncontact measurement methods are based mainly on photoelectric, electromagnetic, and other technologies and not need establish contact with an object during measurement. Typical noncontact measurement methods include laser triangulation, ultrasonic measurement, eddy current methods, and machine vision methods (Ye \& Dong, 2019; Bao \& Li, 2019).

With the continuous development of computer vision technology and image acquisition equipment, visionbased displacement and vibration monitoring methods have been used in civil engineering for more than 10 years

*Corresponding author. E-mail: zhixiangzhou@szu.edu.cn 
and verified in practical engineering applications (Xu \& Brownjohn, 2018; Feng et al., 2015; D. Feng \& M. Q. Feng, 2017a, 2017b; Dong et al., 2018; Chu et al., 2019; Jiang et al., 2020). Owing to their numerous advantages, such as long-distance, noncontact, high precision, time and labor efficiency, multipoint monitoring, and so on, such methods have attracted increasing attention from researchers and engineers (Ye et al., 2016b). These methods mainly perform target tracking on test structure videos taken by a camera to obtain the movement trajectory of a measurement point in the image and determine the displacement information of a structure through the geometric relationship between the image and the real world. A camera is erected at a fixed point away from the test structure, thereby eliminating the need for contact displacement monitoring methods to install fixed support points on the structure. In addition, because the field of view of a camera can cover multiple measurement areas on a structure, achieving multipoint measurements at a low cost is easy.

Typical visual vibration measurement methods include those based on optical interference, target tracking, and digital image correlation. Based on the principles of close-range digital photogrammetry and computer vision, Chang and Ji (2007), Ji and Chang (2008) proposed a video measurement technique for measuring the vibration response of three-dimensional structures in the laboratory. Meanwhile, Ho et al. (2012) proposed an infrastructure dynamic displacement measurement method based on multipoint vision. Moreover, Martins et al. (2013) proposed the use of visual technology to measure the three-dimensional displacement of suspension bridges. Based on correlation theory of digital image processing and a multipoint template matching algorithm, Ye et al. $(2013,2016 a)$ proposed a computer vision-based longdistance noncontact distributed structural displacement monitoring method for long-span bridges, which realized the onsite structural displacement monitoring of distance in kilometers. Zhao et al. (2019) successfully combined support vector filtering and Kanade-Lucas-Tomasi (KLT) tracking method to identify the dynamic displacement of a bridge tower structure. The support vector filtering method first selects a rough area then uses KLT to achieve accurate tracking while exhibiting robustness and accuracy. Computer vision-based modal parameter visual recognition methods have become research hotspots in recent years. Camera measurements without structural surface pretreatment can directly extract structural modal parameters. This technology mainly combines phasebased optical flow calculations (Fleet \& Jepson, 1990) and video motion amplification technology (Wadhwa et al., 2013) processing methods. Chen et al. (2015) of MIT successfully measured the modal parameters of an antenna rod using video motion amplification technology. Sarrafi et al. (2017) used phase-based video motion amplification technology to measure the modal parameters of a fan blade, such as vibration mode and frequency, and compared them with those obtained by an accelerometer, thereby showing the satisfactory accuracy of the method.
Yang et al. (2017a) identified the modal parameters of a laboratory four-layer frame structure with high accuracy and discussed the modal parameters under several conditions, such as when the video frame rate does not meet the Shannon sampling theorem, the possibility of recognition (Yang et al., 2017b), and the establishment of a highly realistic video motion model for structural dynamic analysis that can replace a finite element model (Yang et al., 2018a). Specifically, accurately identifying structural damage is difficult for the traditional structural damage identification method, which is based on modal parameters, owing to limited vibration mode points. Moreover, computer vision technology can provide a simple and practical method for obtaining the vibration mode of dense measurement points, which demonstrates accurate damage identification, thereby providing a solid foundation. $\mathrm{Li}$ et al. (2011) proposed a structural damage identification method based on fractals, which requires dense vibration mode data. Furthermore, Yang et al. (2018b) used a fractal method to identify structural damage based on computer vision to obtain dense vibration mode data.

In addition, the mobile measurement method has been developed in recent years. The available measuring points get increased in space by using on-board sensors to perform indirect measurements. The obtained signal needs to be post-processing to separate the vehicle motion and the bridge response (Matarazzo \& Pakzad, 2018; Eshkevari et al., 2020; Yang et al., 2004). The separation method is studied respectively, and the bridge modal parameters are obtained, including high-resolution mode shapes and modal frequencies in various orders. The separation method requires the information of moderate road roughness and refined control on vehicle speed (Malekjafarian et al., 2015), which needs further studies. However, because of its convenience and reusability, it has a very promising application.

At the same time, the method based on the analysis of time-domain signal of vision-based structural modal identification, the modal parameters, including high-definition mode shapes, have been obtained directly (Eshkevari et al., 2019). The research has achieved good results for small structures in the laboratory. However, it cannot decompose modal using time histories directly since there is no guarantee that partial mode shapes are orthogonal. That means it cannot be used in the method of multiple fields of views.

The summary of the aforementioned studies reveals that displacement and vibration monitoring using machine vision is widely studied and applied in engineering. However, several problems remain in the method, that is: 1) few measuring points exist in the displacement tracking method through target points or KLT feature points, and 2) the measurement accuracy and the size of the field of view are mutually restricted when applying vision-based technologies. To improve the accuracy, the shooting area is usually zoomed in by using a telephoto lens to increase resolution, but this solution reduces the available field of view. This problem becomes more serious when applying 


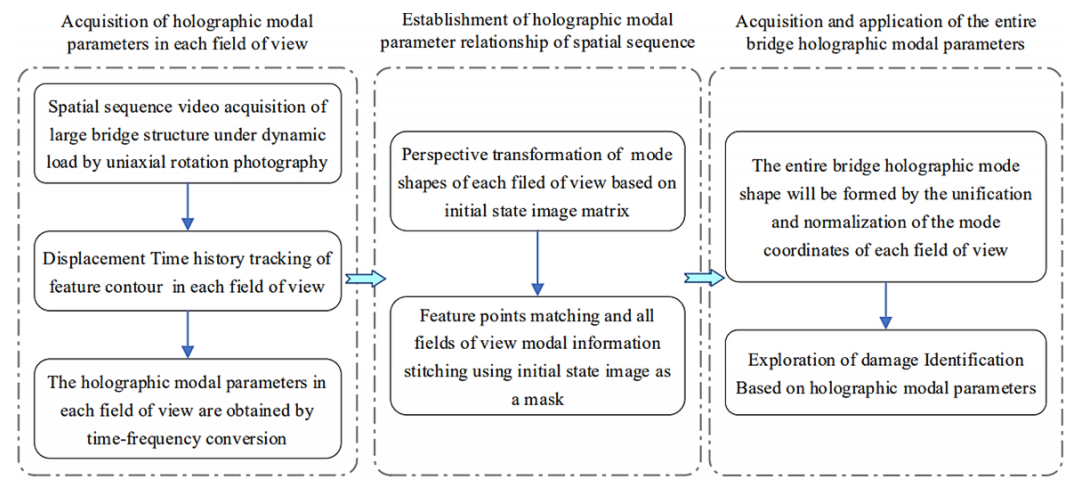

Figure 1. General idea and flow chart

vision-based technologies to a large-span bridge. Therefore, in view of these problems, this study proposes the use of fixed-axis rotation photography, which can record videos of a large bridge structure from different view areas; obtain the time history displacement of a main beam, with the low edge contour as the target; unify the modal parameters of each view area with the same coordinates and match them according to the same name pixel points; and synthesize the modal parameters of the entire bridge. The general idea and process of this paper are shown in Figure 1. According to the method proposed in this study, the number of measuring points can be increased significantly, and dense full-field dynamic parameters can be obtained, which reflect the sensitivity of damage identification to location changes in a low-order mode. Damage identification performance can be improved through the use of modal information, especially when high-order mode information is difficult to obtain.

\section{Theory}

The mode shape and frequency of a structure depend on $\boldsymbol{M}$ and $\boldsymbol{K}$. In the experiment discussed in this paper, the material characteristics of the experimental object remain invariable, and the structural stiffness $K$ is changed to capture the modes and change rule of the structure and to lay a foundation for the subsequent damage identification process. The natural frequency $\omega_{i}$ is a global feature of the structure, which means that it is consistent everywhere in the target object. However, the amplitude and phase of the structural mode sharp $\phi_{i}$ will change in space; thus, the vibration frequency characteristic can be obtained by observing the vibration characteristics of any part of the target object, and the change in structural stiffness in space can be obtained by observing the mode shape. According to the current research, contact and noncontact sensors are limited by input costs, field installation conditions, and technical means, and dynamic parameters, such as frequency and mode, are obtained through limited measuring points. The more the measurement points, the better the damage identification effect. A simple support beam is taken as an example to illustrate the characteristics described above.

\subsection{Eigenmodes analysis}

The beam is simplified into a concentrated mass system supported by a spring and damper for modal analysis, as shown in Figure 2. The dynamic differential equation of the system can be expressed as

$$
\boldsymbol{M} \ddot{x}(t)+\boldsymbol{C} \dot{x}(t)+\boldsymbol{K} x(t)=f(t),
$$

where, $\boldsymbol{M}$ represents the mass matrix, $\boldsymbol{C}$ is the viscous damping matrix, and $\boldsymbol{K}$ is the stiffness matrix. $x, \dot{x}$, and $\ddot{x}$ represent the vectors for displacement, velocity, and acceleration of each point on the simple support beam, respectively. Assuming sinusoidal solutions to Eqn (1), the eigenmodes of this system are the orthogonal solutions to the generalized eigenvalue problem given by $\boldsymbol{K} \phi_{i}=\omega_{i}^{2} \boldsymbol{M} \phi_{i}$. The set of eigenvectors or eigenmodes $\phi_{1} \ldots \phi_{n}$ define a modal matrix $\boldsymbol{\Phi}$ shown in Eqn (2) which diagonalizes the mass and stiffness matrices into modal masses $\boldsymbol{m}_{i}$ and modal stiffnesses $\boldsymbol{k}_{i}$.

$$
\begin{aligned}
& \boldsymbol{\Phi}=\left[\phi_{1}, \phi_{2} \ldots \phi_{N}\right], \\
& \boldsymbol{\Phi}^{T} \boldsymbol{M} \boldsymbol{\Phi}=\operatorname{diag}\left(\boldsymbol{m}_{i}\right), \\
& \boldsymbol{\Phi}^{T} \mathbf{K} \boldsymbol{\Phi}=\operatorname{diag}\left(\boldsymbol{k}_{i}\right) .
\end{aligned}
$$

The matrix $\boldsymbol{\Phi}$ defines modal coordinates $\boldsymbol{q}(t)$ where $\boldsymbol{u}(t)=\boldsymbol{\Phi} \boldsymbol{q}(t)$. In these modal coordinates, the equations of motion are decoupled into single-degree-of-freedom systems defined by modal masses $\boldsymbol{m}_{i}$, damping $\boldsymbol{c}_{i}$, stiffnesses $\boldsymbol{k}_{i}$, and forces $\boldsymbol{f}_{i}(t)=\phi_{i}^{T} \boldsymbol{f}(t)$. Under the common assumption of Rayleigh damping, modal damping can be expressed as $\boldsymbol{c}_{i}=\alpha \boldsymbol{m}_{i}+\beta \boldsymbol{k}_{i}$.

In an undamped system, the natural frequency is $\omega_{i}=\sqrt{\boldsymbol{k}_{i} / \boldsymbol{m}_{i}}$, thereby giving the decoupled equation of motion for each mode as

$$
\ddot{\boldsymbol{q}}(t)+2 \xi_{i} \omega_{i} \dot{\boldsymbol{q}}(t)+\omega_{i}^{2} \boldsymbol{q}(t)=\frac{f_{i}}{\boldsymbol{m}_{i}},
$$

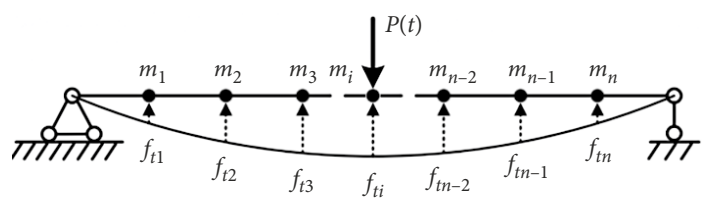

Figure 2. Ideal model of concentrated mass for simply supported beams 
giving the modal damping factor

$$
\xi_{i}=\frac{\boldsymbol{c}_{i}}{2 \boldsymbol{m}_{i} \omega_{i}}=\frac{1}{2}\left(\frac{\alpha}{\omega_{i}}+\beta \omega_{i}\right) \text {. }
$$

Then, the unit impulse response for the $i$ th mode can be obtained by solving Eqn (5).

$$
h_{i}(t)=\left(\frac{e^{-\xi_{i} \omega_{i} t}}{\boldsymbol{m}_{i} \omega_{d i}}\right) \sin \left(\omega_{d i} t\right),
$$

where the damped natural frequency is $\omega_{d i}=\omega_{i} \sqrt{1-\xi_{i}^{2}}$. Taking the Fourier transform of the unit, impulse response $h_{i}(t)$ becomes the convolution:

$$
\begin{aligned}
& H_{i}(\omega)=\left(\frac{1}{\boldsymbol{m}_{i} \omega_{d i}} \frac{\xi_{i} \omega_{i}}{\xi_{i}{ }^{2} \omega_{i}{ }^{2}+\omega^{2}}\right) * \\
& \left(\frac{\delta\left(\omega-\omega_{d i}\right)-\delta\left(\omega+\omega_{d i}\right)}{i}\right) .
\end{aligned}
$$

Therefore, the transfer function of a single mode is the convolution of the spike of the resonance frequency with the Lorentz distribution (the Fourier transform of the exponential decay), whose width depends on the mode frequency and damping. The time spectrum obtained by the broad-spectrum excitation is presented as the transfer function of the target, and the damping of the target can be derived by observing the width of the resonant peak of the time spectrum. The effects of damping can be observed through the motion spectrum, or the Lorentz window is used to match and identify the motion spectra.

The natural frequency of an object is a global characteristic and does not change depending on how it is observed. Thus, the time spectrum obtained from different locations is consistent. An object's power spectrum of local motion at different locations can be obtained by using the invariance of frequency $\omega_{i}$ in space. All the coincident peaks in the power spectrum correspond to the natural frequency. Therefore, the position, size, and direction of each pixel are extracted to form the power spectrum of local motion, and the average can be transferred to the global power spectrum.

The amplitude and phase of the time history signals at each point of the simple support beam are obtained by Fourier transform. The amplitude represents the amplitude of motion, whereas the phase indicates the direction of motion. The signals at each point of the corresponding frequency can be calculated using the amplitude and phase, thereby obtaining the modal characteristics of the structure under the corresponding natural vibration frequency. In modal parameters, frequency and damping are the global characteristics of a structure. Theoretically, the frequency and damping of all modes can be obtained from one measuring point (avoiding nodes of each mode). Numerous points must be measured to obtain the mode shape; thus, the mode shape is a local characteristic of a structure. The more measuring points, the more real the mode shape reflected. The traditional measurement method can fit only the mode shape through a limited number of points. From the perspective of health monitoring, several important information is ignored, and detecting structural stiffness changes at an early stage is difficult. According to the Shannon-Nyquist sampling theorem, in the analog/digital signal conversion process, when the sampling frequency $f_{\text {s.max }}$ is more than 2 times the highest frequency $f_{\max }$ in the signal, the sampled digital signal completely retains the information in the original signal. Therefore, generally, to obtain the third natural frequency of a measured object, the arrangement of $2 i+1$, that is, seven measurement points, meets the requirements. However, if additional measurement points are arranged, then natural frequencies can be obtained accurately, and accurate mode shape parameters can be provided through modal damage identification. The concept can be explained through a numerical simulation.

\subsection{Characteristics of dense full-field modal}

A numerical calculation model is established by taking the simple support beam shown in Figure 1 as an example. The beam length is $3 \mathrm{~m}$, the section is rectangular, height $\mathrm{H}=0.01 \mathrm{~m}$, and width $\mathrm{B}=0.15 \mathrm{~m}$. The beam is divided into 24 elements along its length and is a single degree of freedom along the vertical axis, as shown in Figure 3. The material is Q345 steel, and E $=2.1 \times 10^{5} \mathrm{MPa}$. Element damage is simulated by reducing two thirds of the elastic modulus of the material, and the damage condition is shown in Table 1.

Two data acquisition methods are adopted by taking the process of obtaining the first three order dynamic parameters of the simple support beam as an example. One method is based on the Shannon-Nyquist sampling theorem and uses seven measurement points as data sources (limited measure points), as shown in Figure 3. The other method uses all nodes as data sources (dense measure points) to compare and analyze the impact of significant changes in the number of measurement points on the modal parameter analysis. Based on the theory discussed in Section 2.1, the first three frequencies and modes are obtained and regularized according to the maximum displacement.

Table 1. Damage condition

\begin{tabular}{|l|c|c|c|c|c|c|c|}
\hline Damage condition & 1 & 2 & 3 & 4 & 5 & 6 & 7 \\
\hline Damage units & None & 6 & 7 & 8 & 9 & 10 & 11 \\
\hline
\end{tabular}

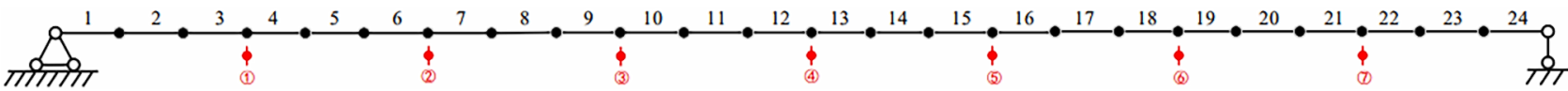

Figure 3. Numerical model 
The first three order frequencies are obtained by dense and limited measuring points, which are $5.85 \mathrm{~Hz}, 16.12$ $\mathrm{Hz}$, and $36.61 \mathrm{~Hz}$. According to Figure 4, the mode shapes of low-order modes obtained by dense and limited measuring points tend to be consistent with their mode shapes under different damage conditions.

However, as mode order improves, mode shapes formed by dense measuring points can better reflect real mode shapes and their shape trends under different damage conditions. At the same time, according to existing research (Dessi \& Camerlengo, 2015), the mode shape cannot quantify damage location and damage degree eas-

a) Mode 1 obtained by limited measuring points

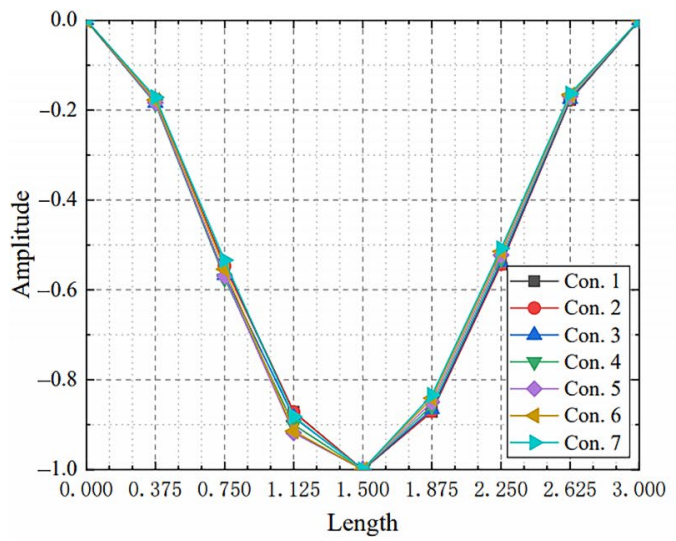

c) Mode 2 obtained by limited measuring points

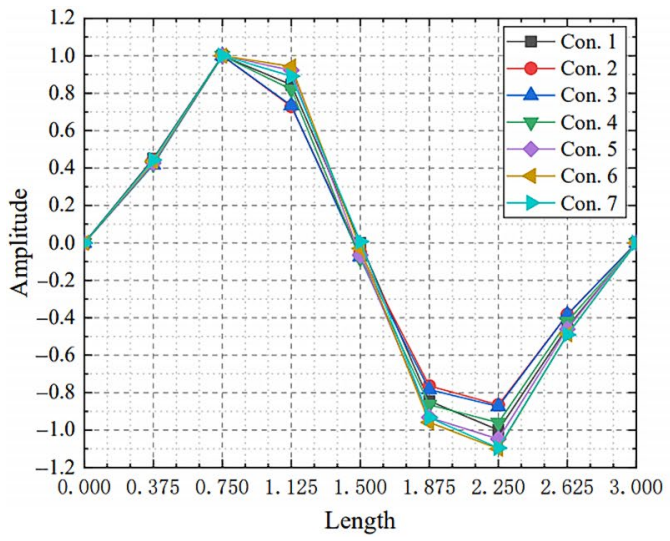

e) Mode 3 obtained by limited measuring points

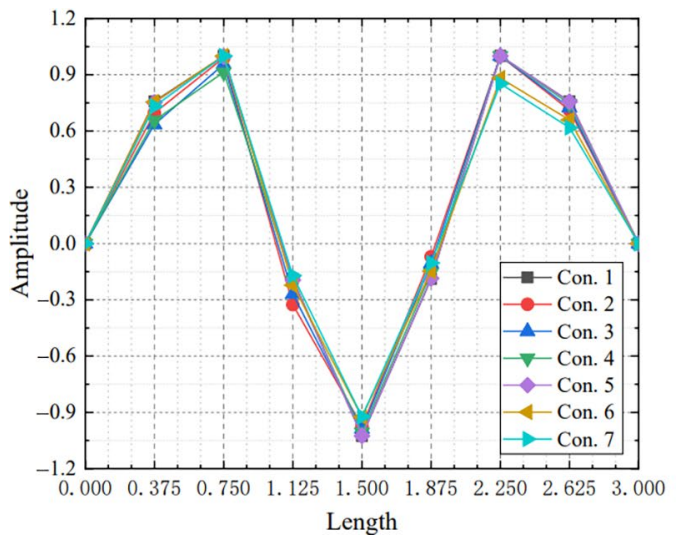

ily and is rarely employed directly in damage identification. However, the curvature mode or flexibility mode obtained through transformation has a satisfactory effect on damage identification. The displacement modes of each node are first calculated by taking the curvature mode as an example, then the curvature mode matrix is obtained through difference approximation.

$$
\varphi_{i}^{\prime \prime}=\frac{\varphi_{i+1}-2 \varphi_{i}+\varphi_{i-1}}{l_{i-1} l_{i}},
$$

where $\varphi_{i}(x)$ represents displacement modes, $i$ is the $i$ th measurement points, and $l_{t-1}$ is the distance between two

b) Mode 1 obtained by dense measuring points

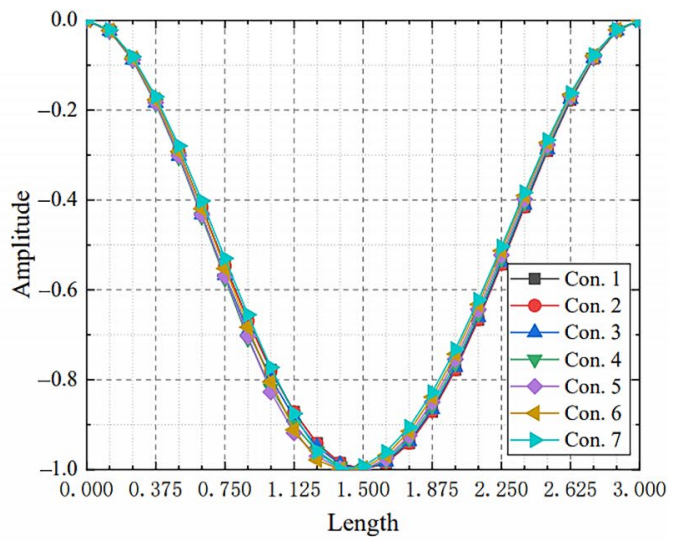

d) Mode 2 obtained by dense measuring points

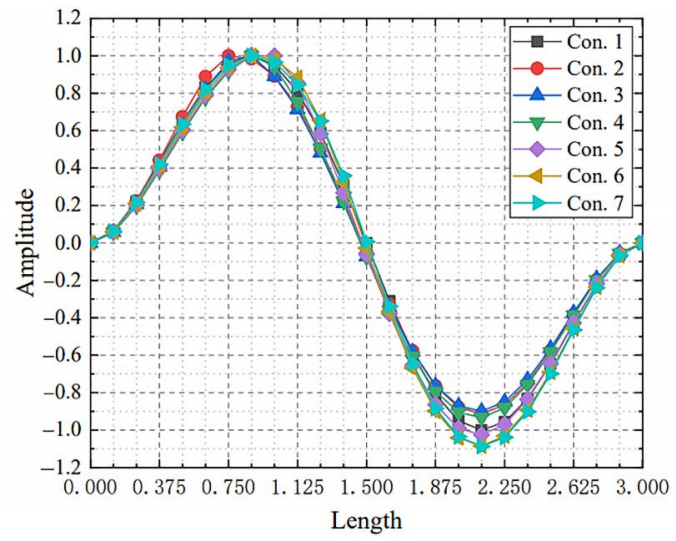

f) Mode 3 obtained by dense measuring points

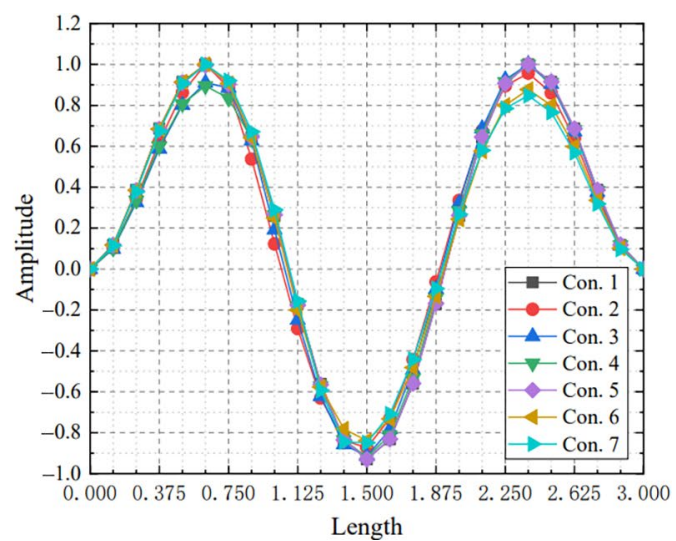

Figure 4. Comparison of modes obtained by dense and limited measuring points 
adjacent measuring points $i-1$ and $i$. The modal curvature difference $\Delta$ before and after damage is

$$
\Delta=\varphi_{u}^{\prime \prime}-\varphi_{d}^{\prime \prime},
$$

where, $\varphi_{u}^{\prime \prime}$ and $\varphi_{d}^{\prime \prime}$ represent the modal matrix of the curvature before and after damage, respectively, obtained through difference calculation:

$$
\delta=\max |\Delta| .
$$

Structural damage identification can be realized by using the property of the maximum value $\delta$ of the modal curvature change vector, and the degree of damage can be reflected by its size.

According to the results, the curvature difference of each node in each damage and undamaged condition in Mode 1 is taken as an example to illustrate the results. As shown in Figure 5a, the curvature mode difference of conditions 2 and 3 is very small, and in conditions 5 and 6 , the node values are basically the same. The relationship between the damage and change in the damage condition is not clear; thus, identifying the damage on this basis is difficult. From Figure 5b, the initial location of the damage is apparent (node number 12 mutations), and with the damage position extending continuously to the left, the abrupt position of the curvature difference of the left node also shifts to the left, thereby showing the correlation in the damage position change and reflecting the sensitivity of damage identification. Meanwhile, highly dense measuring points can be predicted, which are sensitive to damage identification. A self-anchored suspension bridge is taken as an example in the following section to explain how highly dense detection points can be achieved and dense full-field dynamic parameters can be obtained.

\section{Test overview}

\subsection{Test object and data acquisition scheme}

Based on the previous study of our research team (Wang et al., 2016; Shao et al., 2020; Deng et al., 2020), a 1:30 model of the Taohuayu Yellow River Bridge is constructed. A total of 52 C 30 concrete deck slabs $(1.16 \times 0.45 \times 0.2 \mathrm{~m})$ are prepared and laid on a steel box girder to simulate vehicles on the bridge and serve as counterweights.

The test vehicle is a flat car made from a $10 \mathrm{~cm}$-thick steel plate, which can increase the counterweight according to the requirements of the test content. Meanwhile, to ensure that the test vehicle does not deviate during the driving process, channel steel is laid on the bridge deck as the guide rail of the test vehicle, as shown in Figure 6.

Every pixel can be used as a displacement sensor to monitor structures. However, many tests only obtain the motion response of the critical structural parts (Feng et al., 2017; Dong et al., 2019). In this study, a high-speed camera system, proposed in previous work (Deng et al., 2020), was adopted as a noncontact remote bridge intelligent sensing holographic monitoring system to obtain the structural dynamic response. The main components

a) Curvature modal difference of Mode 1 obtained by limited measuring points

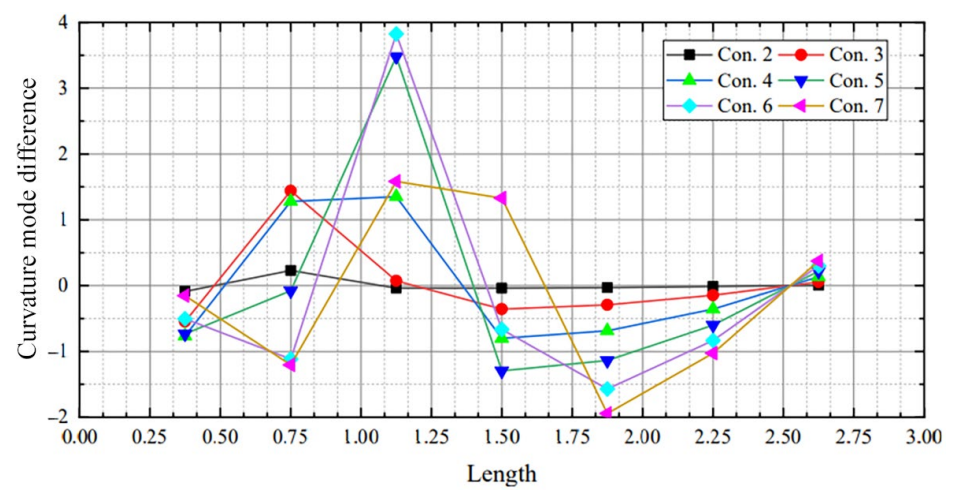

b) Curvature modal difference of Mode 1 obtained by dense measuring points

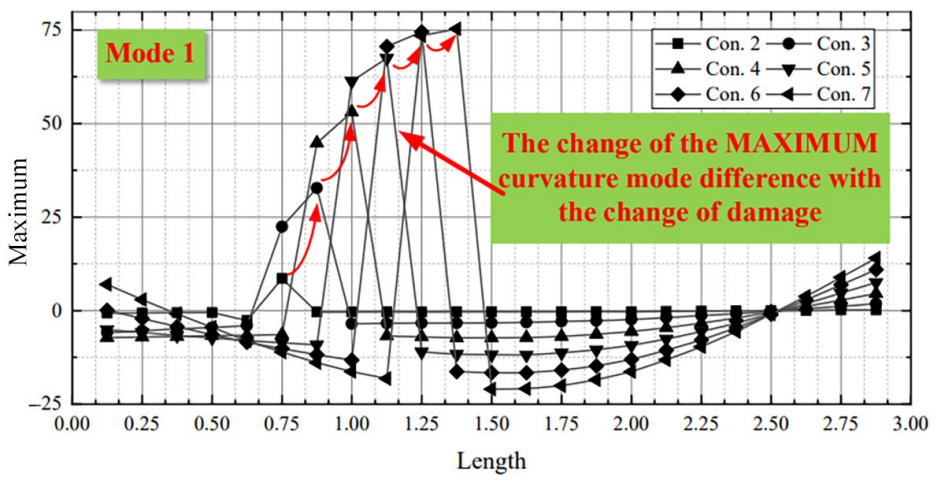

Figure 5. Comparison of mode difference obtained by dense and limited measuring points 
include holographic vision sensor, PTZ, and control system, it is set up $5 \mathrm{~m}$ from the test bridge facade. The fixed angle rotation of the camera is controlled by a computer to realize the acquisition of the fixed-section image of the test bridge at a fixed position, as shown in Figures 7 and 8. In this experiment, according to the previous research results (Deng et al., 2020), the entire bridge is divided into six visual fields to collect image information. The environmental action, vehicle load, and structural state are known and controllable variables in the test. When a holographic visual sensor is used to monitor the holographic shape of the test bridge under various damage conditions, the temperature, illuminance, and humidity in the laboratory environment will have a certain impact on the test results. To obtain relatively stable original data under various damage/action conditions, the test is performed at 9:00 a.m., with good light intensity and relatively constant tempera- ture and humidity under relatively constant ideal indoor environment conditions with minimal disturbance, from July to December 2019, and recorded as the environmental label information.

To improve accuracy as much as possible, the image acquisition time of each field of view is set to one minute. The mechanical structure requires sufficient time to eliminate vibrations when the platform changes from view $i$ to view $i+1$. Although extremely small, the vibrations cannot be ignored in the video. After the onsite test, the platform needs to stand for 5 minutes to eliminate the influence of the vibrations. At the same time, accelerometers were installed on the pedestal and platform to collect the vibration signal of the platform in the process of rotation, which is shown in Figure 9. In the subsequent processing of the time history signal obtained by a high-speed camera, the signal is used as white noise for denoising.



Figure 6. Reduced-scale model

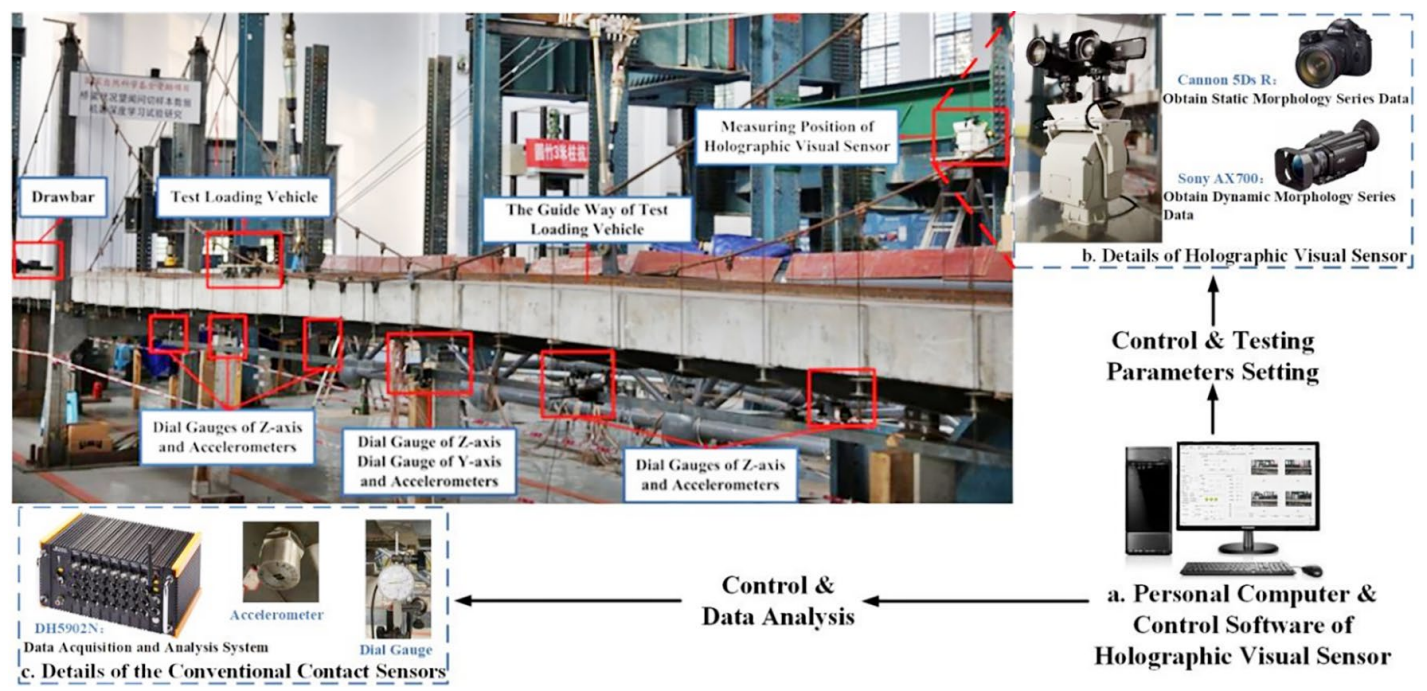

Figure 7. Experiment site layout

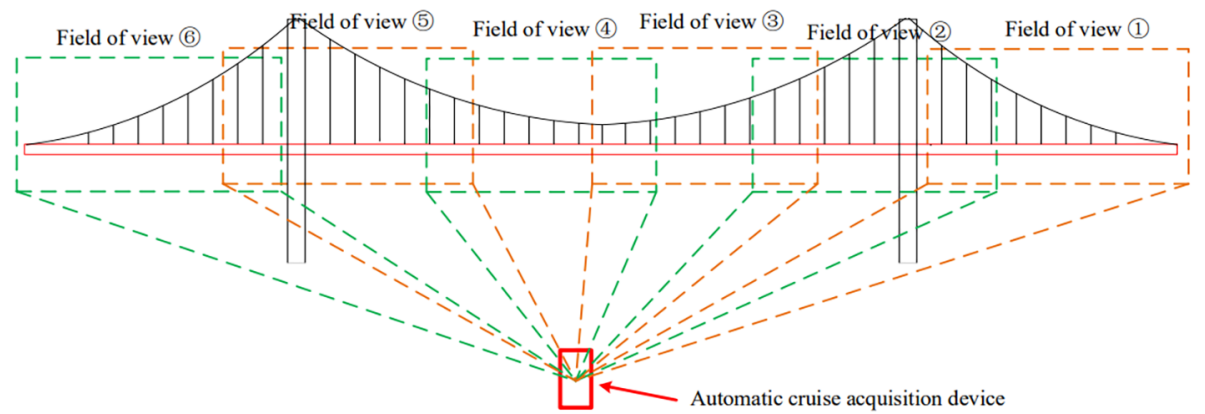

Figure 8. Principle of image collection 


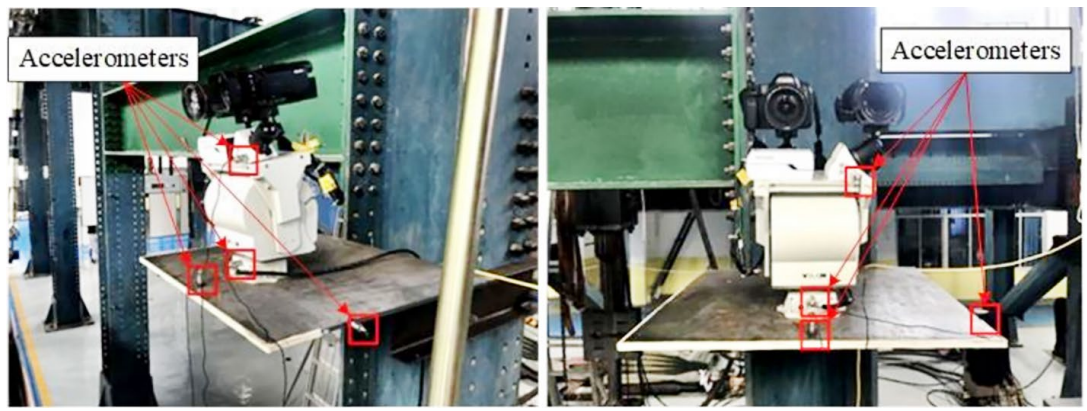

Figure 9. The layout of accelerometers

Given that the scale ratio of the test bridge to the actual bridge is 1:30 in the longitudinal length, the length of the test bridge along the bridge length direction is $1 / 30$ the length of the actual bridge. To simulate a vehicle passing over the actual bridge at a speed of approximately $80-100 \mathrm{~km} / \mathrm{h}$, controlling the speed of the test vehicle to approximately $0.74-0.93 \mathrm{~m} / \mathrm{s}$ through the traction motor is necessary. Under the condition that the test vehicle runs continuously back and forth on the test bridge, the cruising fixed point of the six fields of view is set in the holographic detection system. To ensure adequate constraints between the fields of view and those for the unity and synthesis of subsequent modal vibration modes, a $20-30 \%$ overlap must exist between the fields of view. As shown in Figure 8, videos that are at least one-minute long are collected for each field of view. A high-speed camera collects seven videos from fields of view 1 to 6 .

To verify the feasibility of the image collection method, 11 dial gauges are arranged along the axis of the bridge to capture the dynamic displacement, while the high-speed camera takes videos of the bridge. The arrangement of the dial gauges is displayed in Figure 10.

Accelerometers are arranged along the central axis of the bridge and bridge tower. The acceleration signal of the bridge is collected by the accelerometers and high-speed camera. A total of 14 accelerometers are arranged, 12 of which are placed along the central axis of the main beam.
One accelerometer is arranged in each of the two main towers. Accelerometer Nos. 6 and 7 measure the vertical vibration and transverse vibration at the midspan position, respectively; accelerometer Nos. 13 and 14 measure the vibration in the longitudinal direction of the two main towers; and the other accelerometers measure the vertical vibration. The arrangement of the accelerometers is shown in Figure 11.

The method proposed in this paper collects the image of the bridge facade. In theory, only the vertical degrees of freedom are calculated, and the out of plane degrees of freedom are not included. The suspension bridge is symmetrical along the central axis, and the damage conditions are set symmetrically along the central axis without considering the out of plane changes. Therefore, the dial indicator and accelerometer are arranged along the central axis of the test bridge.

\subsection{Test content}

To provide additional samples for data analysis, the characteristics of the bridge structure behavior can be monitored by the holographic visual sensor. The following two conditions are proposed for collecting the test bridge structure deformation data: (1) Under the condition of a nondestructive structure, the running speed of ordinary vehicles is simulated by adding different weights (total weight of vehicle and counterweights are $25 \mathrm{~kg}$ and $50 \mathrm{~kg}$ );



Figure 10. Arrangement of dial gauges

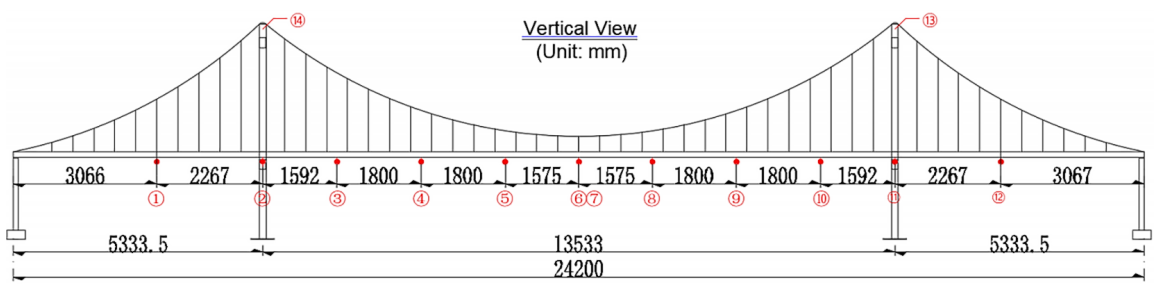

Figure 11. Arrangement of dial accelerometers 


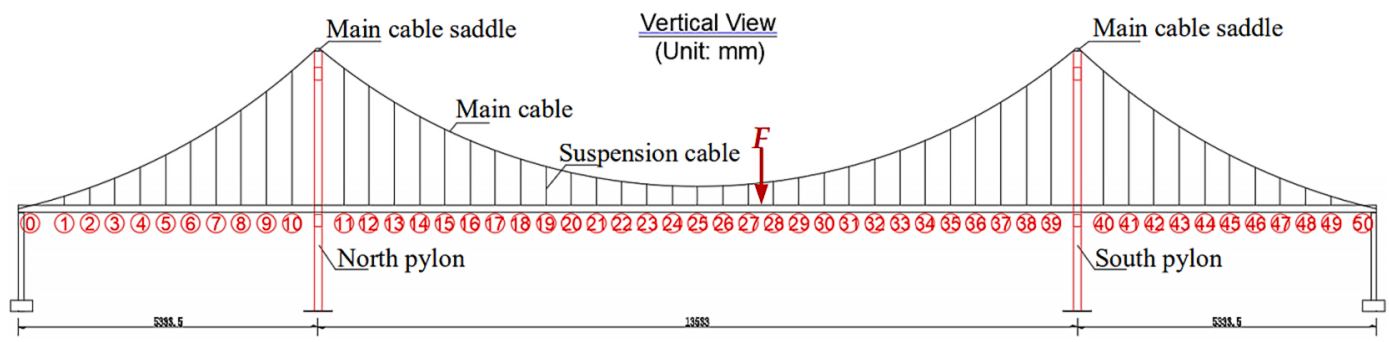

Figure 12. Serial number of suspension cables

Table 2. Test condition and measurement method

\begin{tabular}{|c|c|c|c|c|c|c|}
\hline \multirow{2}{*}{$\begin{array}{c}\text { Serial } \\
\text { number }\end{array}$} & \multicolumn{3}{|c|}{ Test condition } & \multicolumn{3}{c|}{ Measurement methods } \\
\cline { 4 - 7 } & Excitation type & Type & Quantity & Traditional measurement methods & Visual measurement method \\
\hline 1 & \multirow{2}{*}{ Test vehicle } & Dynamic load & $25 \mathrm{~kg}$ & Accelerometer & Dial gauge & Holographic visual sensor \\
\cline { 4 - 7 } & & $50 \mathrm{~kg}$ & Accelerometer & Dial gauge & Holographic visual sensor \\
\hline 3 & \multirow{2}{*}{ Force hammer } & $\begin{array}{c}\text { Single point } \\
\text { force }\end{array}$ & $300 \mathrm{KN}$ & Accelerometer & Dial gauge & Holographic visual sensor \\
\cline { 5 - 7 } & & $600 \mathrm{KN}$ & Accelerometer & Dial gauge & Holographic visual sensor \\
\hline 4
\end{tabular}

(2) The displacement and acceleration data of the test bridge are collected by applying different single-point excitation forces, that is, $300 \mathrm{kN}$ and $600 \mathrm{kN}$, in the same position, as shown in Figure 12. The test condition design is presented in Table 2 .

In order to ensure that the load corresponding to each test condition is consistent, three measures are mainly adopted: (1) a variable-speed motor is used as the traction device of the test vehicle, and the control speed of the test vehicle is adjusted to be consistent in all test conditions; (2) the deck of the test bridge is equipped with a running track to ensure that the driving path of the test vehicle is consistent; (3) the pavement material with the surface of polyester is used to avoid bumping; to keep the level of modal excitations constant along the bridge length.

\section{Dense measuring point displacement monitoring method}

In this study, the motion of a bridge structure in a single field of view collected by a holographic visual sensor belongs to small motions according to the machine vision standard. Although it can lead to motion extraction difficulties, it is relatively easy to analyze. Given that the motion of a structure in a specific state often contains complex nonlinearity, small deformations can be well simulated by a linear system. According to the above assumptions, a method for extracting the natural frequencies and corresponding modes of a bridge structure in a single field of view is established. The modal characteristics of the corresponding bridge structure in a single field of view are obtained by combining the analysis of the characteristic modes with the experimental object in this study.

Figure 13 presents the general framework of the structural displacement monitoring system based on machine vision, which mainly includes four steps and makes corresponding adjustments according to the application scenario, the presence or absence of artificial targets, the implementation algorithm, and so on.

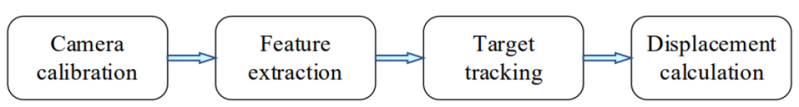

Figure 13. General framework of computer vision-based structural displacement monitoring system

Step 1. The geometric relationship between the image coordinates and world coordinates is obtained through calibration. This step takes advantage of existing research results and thus will not be described in the paper. In this study, the calibration method of Zhang $(2000,2004)$ is used directly and not explained in detail.

Step 2. Selecting appropriate feature points according to the tracking object (in this study, the contour of the lower edge of the main beam) is necessary to obtain the position information of the selected target on the measured structure. As the tracking target of the visual monitoring system, the targets are key to the accuracy of the displacement measurement. The targets are divided mainly into two types. (1) Artificial targets are added on the structure surface to enhance the distinction between the tracking target and the surrounding environment and to improve the tracking accuracy. (2) The image features and texture structure of the structure surface are used as the targets. Given that the objective of this study is to trace the lower-edge contour of the entire test bridge girder to capture a few individual points, the two aforementioned types of markers are not applicable. In this study, the following target tracking methods are proposed according to the characteristics of clear target tracking (the lower-edge contour of the main beam).

The midspan view is taken as an example to illustrate the method for extracting the contour of the lower edge of the main beam of the test bridge feature points. First, the premise is that the position of each view is fixed. To reduce unnecessary calculations and noise, the first frame of the video is extracted and converted into a picture according to the previous research results (Deng et al., 2018) by employing the edge function in MATLAB. Next, the gray- 
scale and contour information of the image is extracted, and the Canny edge detector is adopted for the extraction process. This operator determines the edge points in four steps, that is, smoothing the images with a Gaussian filter, computing the gradient amplitude and direction through finite-difference computing with a first-order derivative, applying no maximum suppression to the gradient amplitude, and using a double threshold to detect and connect the edges.

The Canny edge detector can effectively extract the contours of the bridge structure from the static images collected by the holographic visual sensor. The extracted contours are further processed with MATLAB to decontextualize the contours of useless parts, thereby leaving only the lower-edge contour of the deck slabs to reflect the variation in the structural shape.

Given that the fields of view in the six images are fixed, the contours of the bridge structure are determined through the following method. The six images containing the initial boundary of the bridge structure are taken as the original images. The coordinates of each pixel in the boundary are extracted from the six images. Each pixel is marked in the original images based on these coordinates, thereby revealing the position of the initial boundary. The flowchart of virtual mark point setting of lower-edge contour of main beam is shown in Figure 14a. The manual marking helps suppress noise in the images. In the subsequent target tracking, the contours can be tracked automatically based on the marked pixels, thereby revealing the displacement of the lower-edge contour of the bridge main beam. The comparison between the proposed method and conventional algorithm for feature point extraction is shown in Figure 14b.

Step 3. According to the selected marker points for target tracking, because the structural deformation of the test bridge belongs to small motions in the field of machine vision, first, the K-nearest neighbor classification method (Goldstein, 2003) is used to extract the k-nearest neighbor matching. Next, the best matching is selected through threshold processing. Based on single-strain matrix transformation, the random sampling consistency method (RANSAC) (Matas \& Chum, 2004) is used to remove the abnormal matching.

Step 4. The displacement of each point on the contour line in the image is obtained by comparing the tracked position with the initial position. In addition, the unique information in the image is transformed into the actual displacement in the world coordinate by using the camera calibration information.

According to the above steps, the time history displacement information of dense measuring points of the structural feature contour can be obtained directly, as shown in Figure 15. According to the theory mentioned in Section 1.1, the time history displacement information of dense measuring points can be transformed to more accurate modal information, including frequency and mode shape, than that of limited measuring points. At the same time, according to Section 1.2, the more holographic mode shapes can better identify the damage.

\section{Establishment of modal spatial relations in different fields of view}

As described in Section 1.1, the basic principle of this chapter states that the frequency and mode shape of each stage of the structure are inherent characteristics. Although the two characteristics are obtained from different fields of view, they can form the mode shapes of the entire bridge as long as they are normalized and constrained by the feature points. However, because the data acquisition method used in this study for large-scale bridges is fixed-axis rotation photography, only one field of view involves orthogonal photography, and the rest involves tilt photography.

a) Flowchart of virtual mark point setting of lower-edge contour of main beam

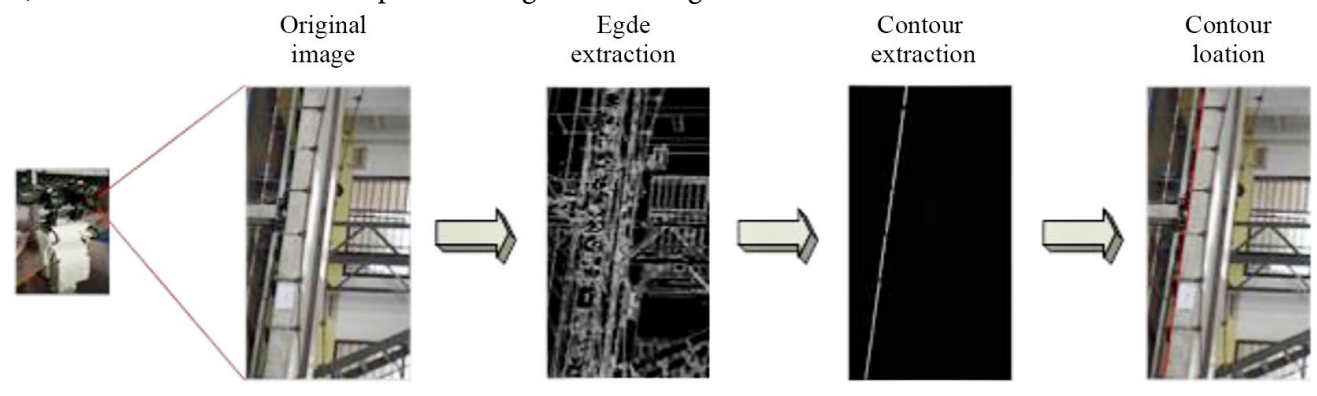

b) The comparison between the proposed method and conventional algorithm for feature point extraction

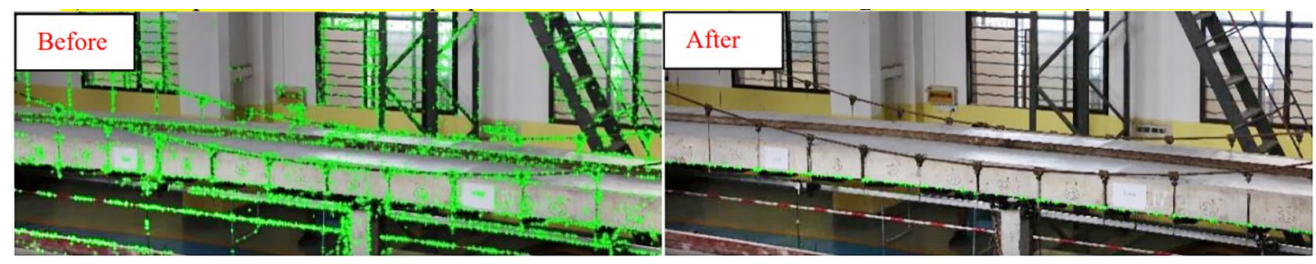

Figure 14. Feature point extraction based on feature contour 




Figure 15. Obtain the modal information schematic diagram of the structure through the image

Thus, according to the method described in Section 3, the amplitude of the time displacement information of each tracked pixel point is not at the same scale even in the same field of view.

Compared with small components, ignoring the difference between the actual size represented by the center pixel and the edge pixel of the image is feasible. However, the object in the video photographed by the method proposed in this study is a large component, the scope of a single field of view is wide, and the closer the camera to the image, the larger the size difference between the center pixel and the edge pixel of the image, which cannot be ignored. In this case, the two actual parallel lines (e.g., the upper contour and lower contour of the bridge slab) will converge to a point in the image, and orthogonality is no longer maintained, which belongs to the distortion generated by the three-dimensional object in the projected image, that is, perspective mapping. To unify the actual size of each pixel in the image, perspective transformation is necessary. However, the video cannot perform perspective transformation directly. Thus, this study first proposes to obtain the amplitude and phase values of each point of the contour of each frequency then form the modal information of each frequency and use the method of perspective transformation to unify the coordinates and finally, match the mode of each field of view with the same name pixel points to form the constraint and synthesize the mode of the entire bridge.

The mode of each field of view is a two-dimensional value without a reference object. Thus, there is no basis to unify the coordinate system of each point. In this study, based on the perspective transformation, the original im- age is used as a mask, and the mode information of each point of the lower-edge contour is added, as shown in Figure 16. The original image is used as the reference, and the perspective transformation is conducted to unify the modal coordinates of each point.

Image geometric transformation refers to the geometric transformation of image pixel position without changing the original image content. It involves basic transformation, including translation, rotation, scaling, reflection, and crosscutting. Perspective transformation and other types of composite transformation are combined through basic transformation, and the image formed by a camera tilted to shoot the target structure on the original imaging plane will be deformed. If the image is mapped to the plane where the target structure is located, which is equivalent to shooting the target structure perpendicularly, then the real shape of the target structure can be obtained. This process is called perspective transformation (Mezirow, 2014), and its mathematical model is shown as Eqn (12):

$$
\left[\begin{array}{l}
x^{\prime} \\
y^{\prime} \\
z^{\prime}
\end{array}\right]=\left[\begin{array}{lll}
a_{11} & a_{12} & a_{13} \\
a_{21} & a_{22} & a_{23} \\
a_{31} & a_{32} & a_{33}
\end{array}\right]\left[\begin{array}{l}
x \\
y \\
z
\end{array}\right],
$$

where $\left[\begin{array}{lll}a_{11} & a_{12} & a_{13} \\ a_{21} & a_{22} & a_{23} \\ a_{31} & a_{32} & a_{33}\end{array}\right]$ is the transformation matrix, the source point is $(x, y, z)^{T}$, and the target point is $\left(x^{\prime}, y^{\prime}, z^{\prime}\right)^{T}$. The image is a two-dimensional object; thus, the value of $z$ of the source coordinate is always 1 . Similarly, the converted image is a two-dimensional object, and the corresponding coordinate is $X=x^{\prime} / z^{\prime}$ and $Y=y^{\prime} / z^{\prime}$.
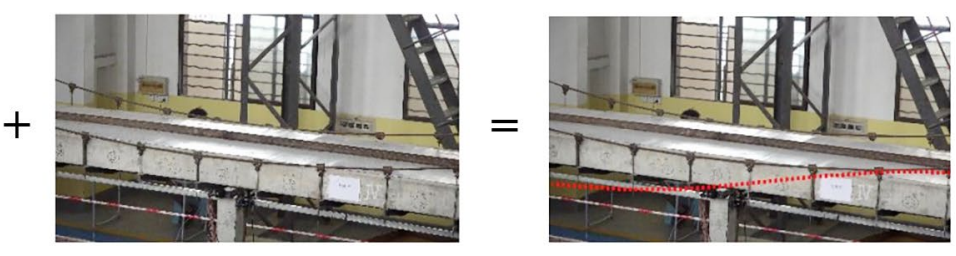

Figure 16. Schematic diagram of mode masked by initial image 
Next, Eqns (13) and (14) can be obtained according to Eqn (12).

$$
\begin{aligned}
& X=\frac{x^{\prime}}{z^{\prime}}=\frac{a_{11} u+a_{21} v+a_{31}}{a_{13} u+a_{23} v+a_{33}} ; \\
& Y=\frac{y^{\prime}}{z^{\prime}}=\frac{a_{12} u+a_{22} v+a_{32}}{a_{13} u+a_{23} v+a_{33}} .
\end{aligned}
$$

According to the principle of perspective conversion, it is known that it belongs to a linear transformation. In the actual projection imaging process, distortion will inevitably occur. If the distortion is not eliminated, the result of the modal perspective conversion will produce an error. Therefore, the deformation correction must be carried out, before calculating the perspective conversion matrix of the initial state image. Here, Zhang's calibration method is also adopted.

Given that the data acquisition method of the intelligent NRS involves the cycle rotation of a fixed axis, the corresponding rotation angle of each visual field is fixed, and the corresponding perspective transformation is conducted for the image collected in each visual field. Moreover, the perspective transformation is performed based on the lower-edge contour of the bridge deck. The trans- formed image retains the complete information of the experimental beam. Although the information of the rest parts is distorted, the deformation of the bridge structure in this field of view is real, as shown in Figure 17.

After the mode shapes of each order obtained from the six fields of view are perspective transformed, the same pixel points of the same order mode shape in the overlapping part between the fields of view can be used for the constraint, as shown in Figure 18. The coordinate points of the mode shapes in the overlapping area are used to normalize and unify the mode shapes coordinates of each view area, then the mode shapes of each order of the entire bridge can be obtained, as shown in Figure 19.

\section{Experimental results and analysis}

\subsection{Frequency extraction}

The results of the dial gauges, accelerometers, and method used in this study (i.e., the holographic visual sensor) are compared. The frequency of any point of the object is the same; thus, the time history curve of the point at the lower edge of the midspan bridge deck (as shown in Figure 20) and power spectrum after Fourier transform can be selected to obtain the first three natural frequencies.
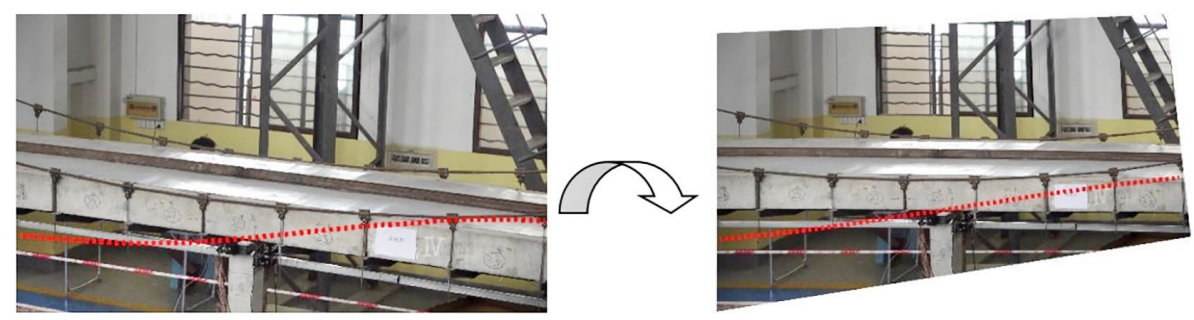

Figure 17. Perspective transformation based on lower contour of bridge deck

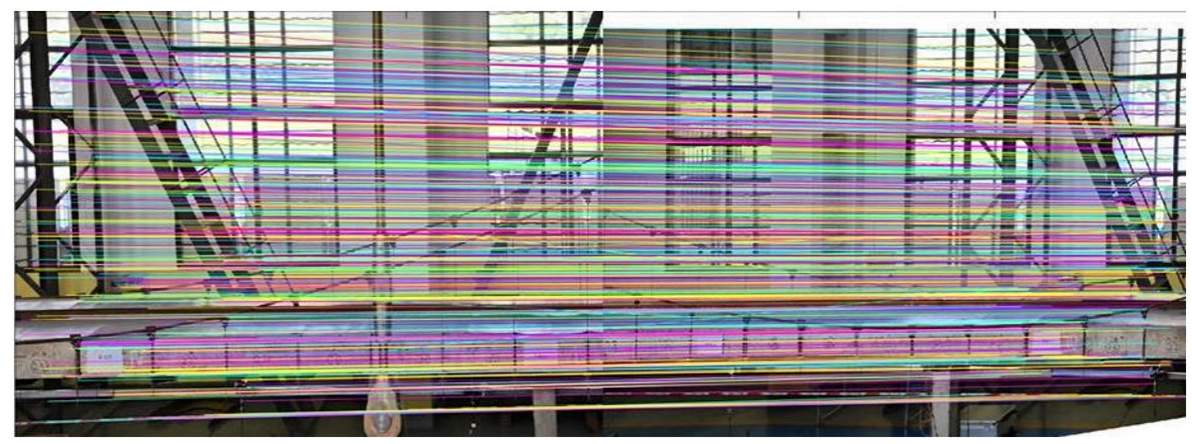

Figure 18. Modal information matching of each field of view using initial state image as mask

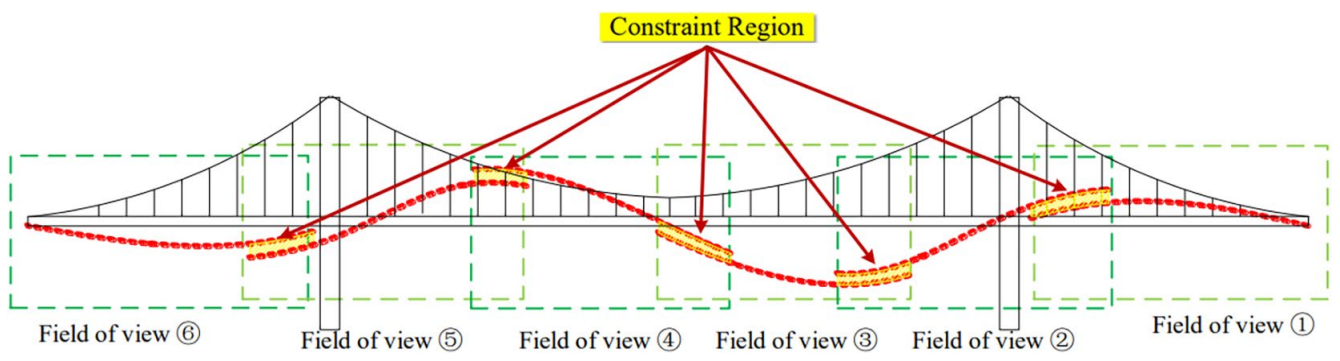

Figure 19. Constraint of modal shapes of fields of view 


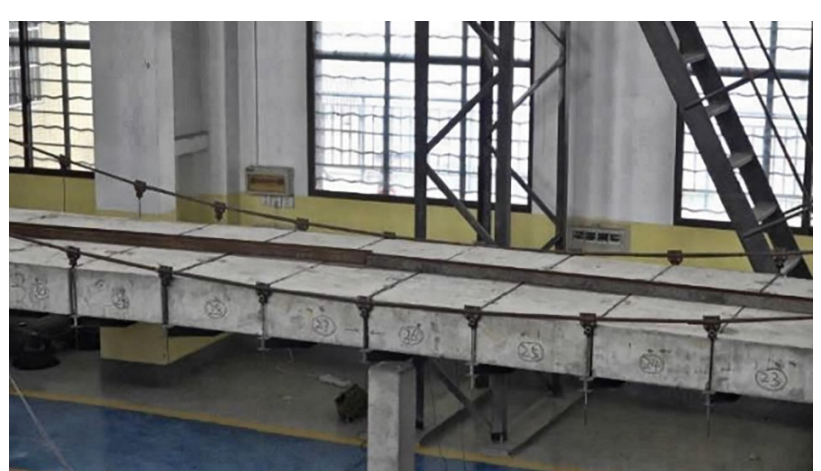

Figure 20. Example view field of frequency and mode extraction

The working conditions of this part of the test are shown in Table 3. First, the test bridge is excited by the motor traction test vehicle, and single-point excitation is conducted with a force hammer. The natural frequency of the test bridge is obtained by the accelerometers, dial gauges, and holographic visual sensor to verify the accuracy of the dynamic signal obtained by the holographic visual sensor.

The time history curve obtained by the holographic visual sensor is compared with that obtained by the dial gauges under conditions $\mathrm{A} 1, \mathrm{~A} 2, \mathrm{~B} 1$, and $\mathrm{B} 2$. The two curves are in good agreement, and the overall trend is the same, as shown in Figure 21.

To further verify the accuracy of the data obtained by the holographic visual sensor, the displacement time history data collected by the holographic visual sensor and accelerometers are used to obtain the first three order natural frequencies of the test bridge under the no damage condition through Fourier transform, as shown in Figure 22. According to Figure 22, the holographic visual sensor

a) Time domain of $\mathrm{A} 1$

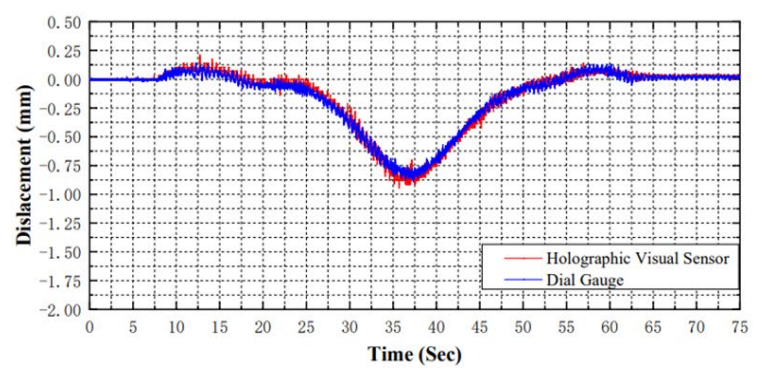

c) Time domain of B1

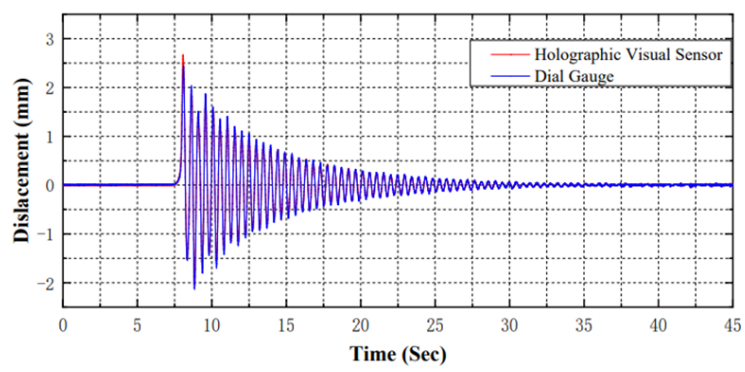

Table 3. Test conditions

\begin{tabular}{|c|c|c|c|}
\hline \multirow{2}{*}{$\begin{array}{c}\text { Test } \\
\text { condition }\end{array}$} & \multirow{2}{*}{$\begin{array}{c}\text { Serial } \\
\text { number }\end{array}$} & \multicolumn{2}{|c|}{ Parameters } \\
\hline & & Test load (kg) & Test speed $(\mathrm{m} / \mathrm{s})$ \\
\hline \multirow{2}{*}{$\begin{array}{l}\text { Driving } \\
\text { excitation }\end{array}$} & A1 & 25 & 0.5 \\
\hline & $\mathrm{A} 2$ & 50 & 0.5 \\
\hline \multirow{2}{*}{$\begin{array}{l}\text { Force } \\
\text { excitation }\end{array}$} & B1 & 30 & 1 \\
\hline & B2 & 60 & 1 \\
\hline
\end{tabular}

can obtain the first three order frequencies well, which is consistent with the frequency value obtained by the accelerometers. The results are shown in Table 4 .

Table 4 shows that after Fourier transform, the time history displacement obtained by the holographic visual sensor and accelerometers is highly consistent with the frequency, and the highest error is $2.8 \%$. Thus, it can be proven that obtaining the dynamic parameters of a structure with a holographic visual sensor is feasible.

At the same time, the results demonstrate that under $60 \mathrm{~kg}$ single-point excitation, the holographic visual sensor and accelerators can obtain the first three order frequencies of the test bridge. However, under $30 \mathrm{~kg}$ singlepoint excitation, the second- and third-order frequency information obtained by the holographic visual sensor after Fourier Transform is not obvious but can be obtained by the accelerometers. This finding means that the amplitude of the $30 \mathrm{~kg}$ single-point excitation is not sufficiently high. However, the accelerometers are adequately sensitive to capture time history information completely, whereas owing to its resolution, the holographic visual sensor loses a part of its amplitude information, thereby resulting in the loss of relatively high-order frequency.

\section{b) Time ldomain of A2}

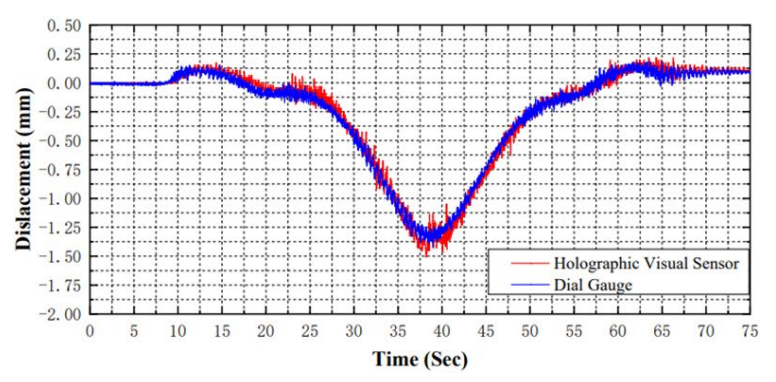

d) Time domain of B2

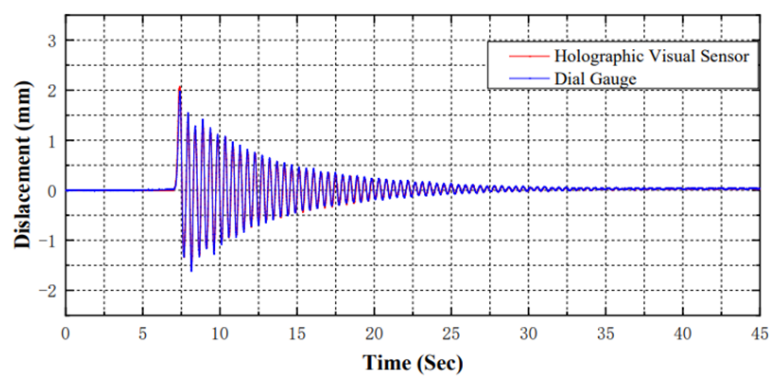

Figure 21. Comparison of displacement extraction between holographic visual sensor and dial gauges 
a) Comparison between measured frequencies of $\mathrm{A} 1$



c) Comparison between measured frequencies of B1

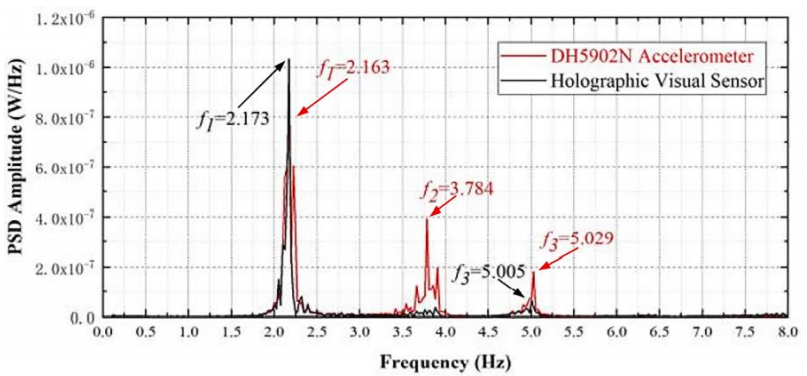

b) Comparison between measured frequencies of A2



d) Comparison between measured frequencies of B2

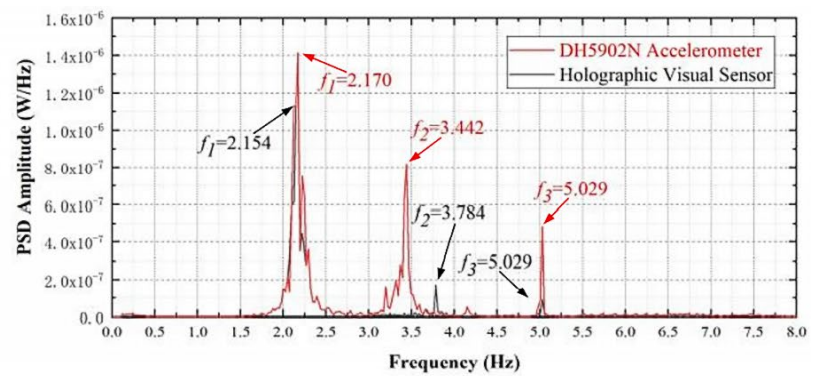

Figure 22. Comparison of frequencies measured by accelerometers and holographic visual sensor

Table 4. Comparison of frequencies obtained by holographic visual sensor and accelerometers

\begin{tabular}{|c|c|c|c|c|c|}
\hline Serial number & condition & order & Holographic Visual Sensor & Accelerometers & Error \\
\hline \multirow{3}{*}{1} & \multirow{3}{*}{ A1 } & First & 2.173 & 2.197 & $1.1 \%$ \\
\hline & & Second & 3.784 & 3.857 & $1.9 \%$ \\
\hline & & Third & 4.907 & 4.883 & $0.5 \%$ \\
\hline \multirow{3}{*}{2} & \multirow{3}{*}{ A2 } & First & 2.112 & 2.100 & $0.6 \%$ \\
\hline & & Second & 3.735 & 3.650 & $2.8 \%$ \\
\hline & & Third & 4.700 & 4.822 & $2.5 \%$ \\
\hline \multirow{3}{*}{3} & \multirow{3}{*}{$\mathrm{B} 1$} & First & 2.129 & 2.173 & $2.0 \%$ \\
\hline & & Second & 1 & 3.784 & 1 \\
\hline & & Third & 5.005 & 5.029 & $0.5 \%$ \\
\hline \multirow{3}{*}{4} & \multirow{3}{*}{ B2 } & First & 2.291 & 2.329 & $1.6 \%$ \\
\hline & & Second & 3.784 & 3.442 & $9.9 \%$ \\
\hline & & Third & 5.029 & 5.029 & 0 \\
\hline
\end{tabular}

The current Sony FDR-AX700 4K HDR HD digital camera is used as the holographic visual sensor. A single-point excitation of $30 \mathrm{~kg}$ is insufficient to excite second- and third-order frequencies, but $60 \mathrm{~kg}$ is adequate. At the same time, Figures 22a and 22b show that under the driving excitation, the participation degree under each order frequency obtained by the holographic visual sensor is the same as that obtained by the accelerators. However, the PSD amplitude under condition A2 with a $50 \mathrm{~kg}$ vehicle weight is extremely obvious, which shows that $50 \mathrm{~kg}$ is suitable as the intensity. Therefore, the equipment limitation and excitation mode should be considered when using this method to collect dynamic parameters at this stage.

\subsection{Mode shape extraction}

According to Section 5.1, the time history information obtained by driving excitation is selected for modal analysis. In this study, the dense full-field measurement is proposed to extract the time history information of all points on the lower-edge contour of the main beam. After Fourier transform, the amplitude and phase corresponding to the first three natural frequencies can be obtained, then the first three order dense full-field mode shapes can be obtained. The acquisition of the amplitude and phase is shown in Figure 23, but the amount of data is too large to display. Therefore, the amplitude and phase of seven points in the midspan view (as shown in Figure 20) of condition A1 are selected as an example to display, as shown in Table 5. 


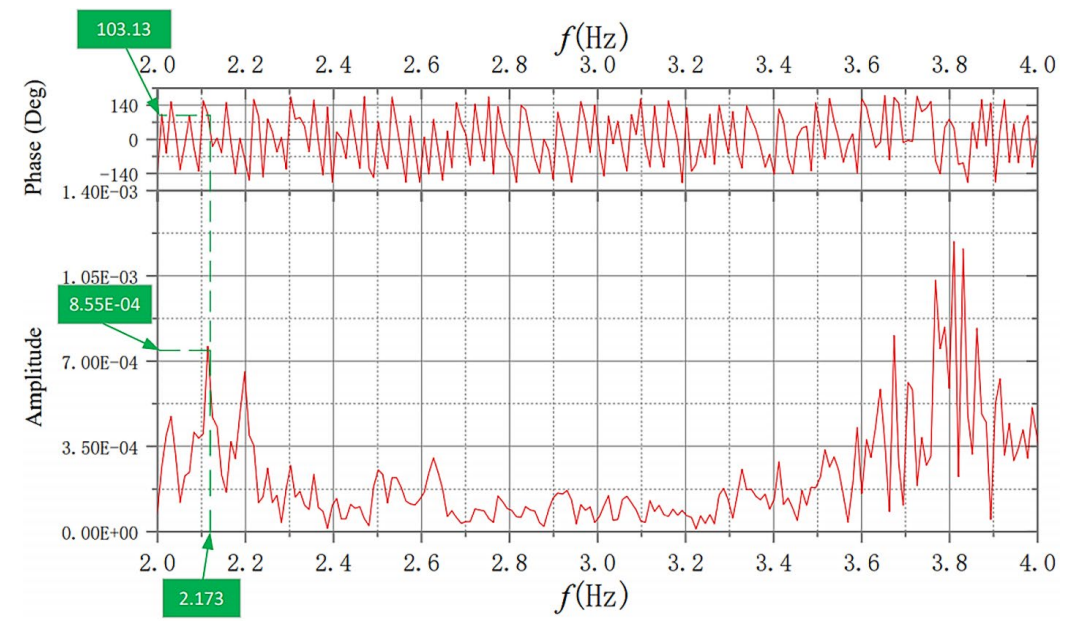

Figure 23. Schematic diagram of obtained amplitude and phase

Table 5. Amplitude and phase of first three order response frequencies

\begin{tabular}{|c|c|c|c|c|c|c|c|c|}
\hline Modal order & Measuring point & 1 & 2 & 3 & 4 & 5 & 6 & 7 \\
\hline \multirow{2}{*}{1} & Amplitude & 0.00239 & 0.00162 & $8.55 \mathrm{E}-4$ & 0 & $8.55 \mathrm{E}-4$ & 0.00162 & 0.00239 \\
\cline { 2 - 9 } & Phase $\left(^{\circ}\right)$ & -90.65 & -21.47 & -6.61 & 0.52 & 103.13 & 141.54 & 121.68 \\
\hline \multirow{2}{*}{2} & Amplitude & 0.00106 & 0.0011 & 0.00112 & 0.00113 & 0.00112 & 0.0011 & 0.00106 \\
\cline { 2 - 9 } & Phase $\left(^{\circ}\right)$ & 81.06 & 151.76 & 46.08 & 179.57 & 40.1 & 124.64 & 121.47 \\
\hline \multirow{2}{*}{3} & Amplitude & 0.0015 & 0.00185 & 0.00207 & 0.00215 & 0.00207 & 0.00185 & 0.0015 \\
\cline { 2 - 9 } & Phase $\left(^{\circ}\right)$ & -67.74 & -63.64 & -155.75 & -115.28 & -24.56 & -59.72 & -55.91 \\
\hline
\end{tabular}

In Table 5, the maximum value of the amplitude of the corresponding measuring point of each mode is used as the normalization factor, and the positive and negative vibration modes of each measuring point are determined by the phase angle $\left(\Phi=0^{\circ} \sim 180^{\circ}\right.$ is positive, and $\Phi=-180^{\circ} \sim 0^{\circ}$ is negative in this study). Given that the phase angle determines only the positive and negative problems of the vibration mode of the measuring point, its numerical value does not play a substantial role in the judgment of the positive and negative problems of the vibration mode. Thus, considering the error of the phase measurement value in the measurement process too much is unnecessary. The mode shape is the ratio of two amplitudes between each measuring point to the selected reference point on the structure to be measured, which is independent of the vibration size of each measuring point. The mode shape can be obtained by comparing the value of each measuring point with the value of the normalization factor, as shown in Figure 24. The figure presents the first three order modes of the main beam in the midspan field of view obtained by the method described in this paper.

To further verify whether the mode shapes obtained by the holographic visual sensor are credible, the mode shapes obtained from the six visual fields are constrained to form the entire bridge mode shape, as described in Section 4 , then compared with the first three modes obtained by the accelerometers, as shown in Figure 25 . The mode shapes and their trend obtained by the two methods are consistent; thus, the mode shapes obtained by the holographic visual sensor are reliable. In this study, a camera is used as a sensor to collect the vibration information of the test bridge to obtain the dense full-field dynamic modal parameters of the structure. Then, the amplitude and phase information of all points of the lower-edge contour of the test bridge in six fields of view is extracted, and through the same name pixel points for normalization and feature matching, the continuous mode shape can be obtained, which can obviously help with damage identification (Yang et al., 2018a).

According to existing research, damage identification with mode shapes requires a high-order mode to show its advantages (Dessi \& Camerlengo, 2015). However, based on the theory mentioned in Section 1, a low-order mode shape obtained by dense measuring points can also reflect sensitivity to damage identification. Substantial intensive measuring point information can be obtained using the full-field girder alignment monitoring method proposed in this study compared with the traditional measuring method. In the test, the suspender was removed symmetrically in the transverse direction to make artificial damage. According to the method described in Section 1, several damage conditions listed in Table 6 are simulated in the laboratory, and the modal curvature difference under each damage condition is obtained separately by the method described in this paper and accelerometers, as shown in Figure 26. 

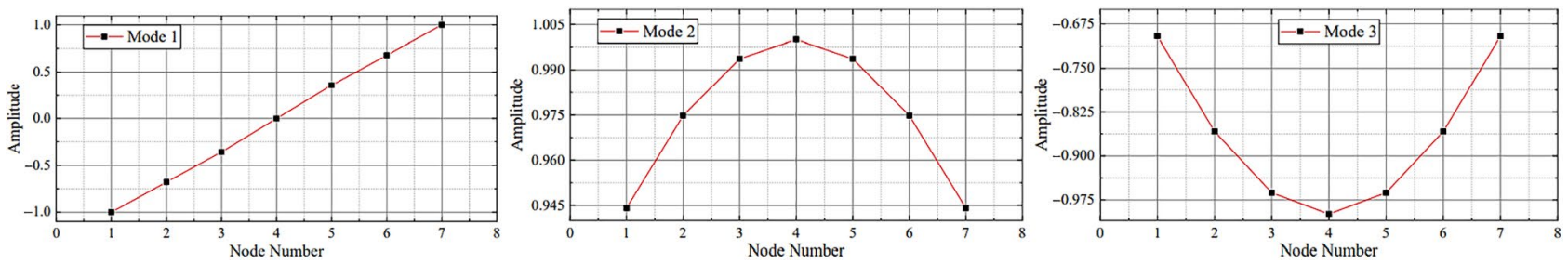

Figure 24. First three order mode shapes of midspan field of view
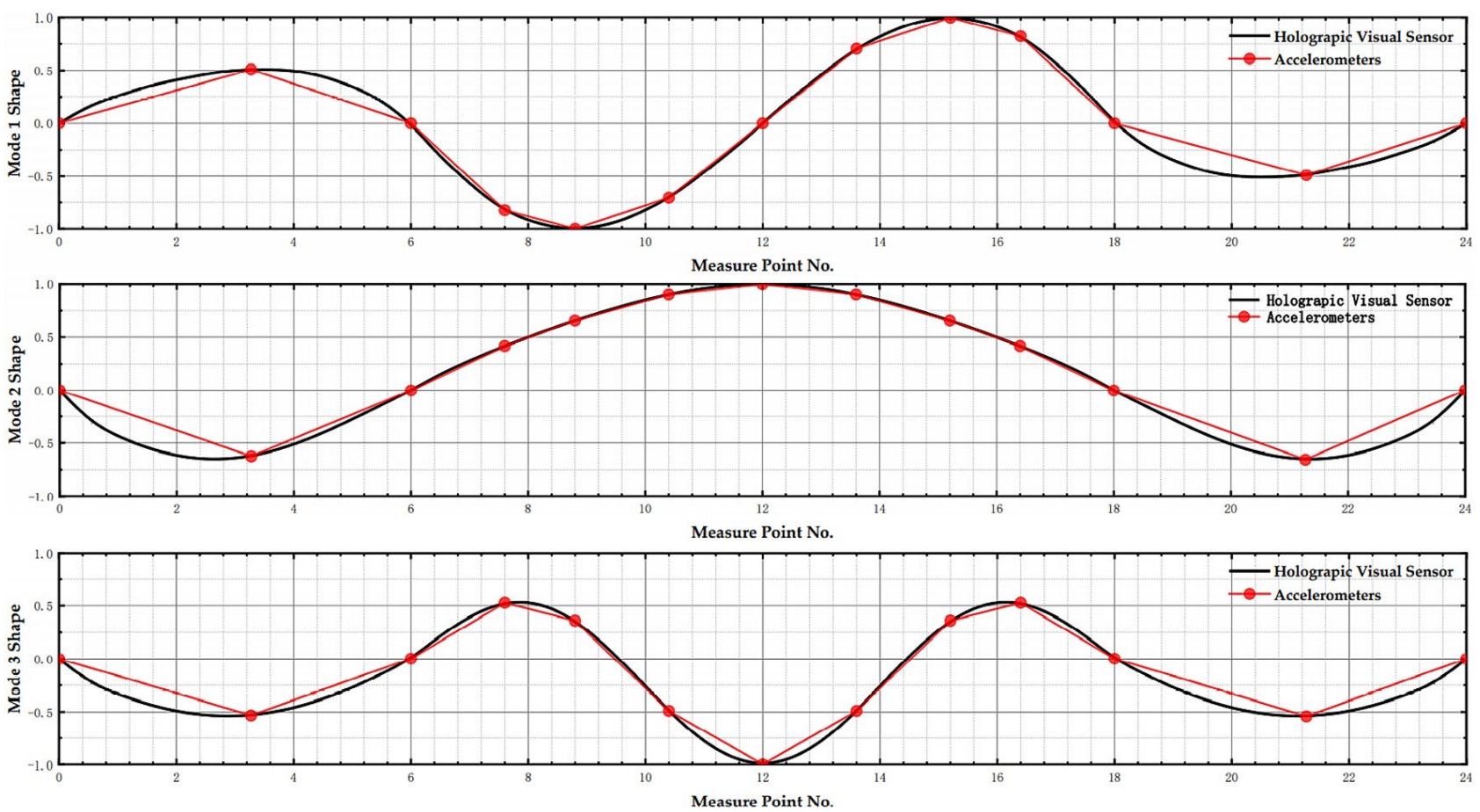

Figure 25. Comparison of mode extraction between accelerometers and holographic visual sensor

Figure 26 shows that the modal curvature difference obtained by the limited measuring points changes under various damage conditions. However, reflecting the changes of the conditions listed in the Table 6 is difficult, and the differences between the modal curvature differences are irregular.

However, Figure 27 demonstrates that the modal curvature difference of the first three order modes under each damage condition obtained by the dense full-field mode experiences a sudden change in the corresponding damage location, and the corresponding sudden change location changes with the different damage locations, which one-to-one corresponds to the damage location.

This finding shows that even in the low-order mode, as long as the dense modal information can be obtained, it can also reflect sensitivity to damage. The advantage of this outcome in practical applications is that a high-order mode is extremely sensitive to damage but difficult to obtain, whereas a low-order mode can be obtained accurately. The dense full-field mode obtained by the technical means proposed in this study can also reflect sensitivity to damage in the low order, which can undoubtedly increase the ease of use of damage identification by dynamic parameters. Although the mutation position does not ex-
Table 6. Suspension cable damage units

\begin{tabular}{|l|c|c|c|c|c|c|}
\hline Damage condition & 1 & 2 & 3 & 4 & 5 & 6 \\
\hline Damage units & 24 & 23 & 22 & 21 & 20 & 19 \\
\hline
\end{tabular}

hibit the largest modal difference, the method proposed in this study can be used to establish long-term monitoring conveniently, and the damage location can be obtained by observing its changes.

\section{Conclusions}

In this study, the dynamic response of a suspension bridge girder under a simulated vehicle load condition is collected using a high-speed camera as a holographic visual sensor. First, virtual marker points are established with the coordinates of the lower-edge contour line of the main beam, and the marker points are tracked by the dense optical flow algorithm to obtain the time history displacement information of each point on the contour line. Second, the dense full-field mode shape and frequency of the main beam are obtained. Finally, relevant dynamic parameters, such as the frequency and mode shape of the entire bridge girder, are identified by establishing the constraints 



Figure 26. Modal curvature difference of first three order modes under different damage conditions obtained by limited point mode shape
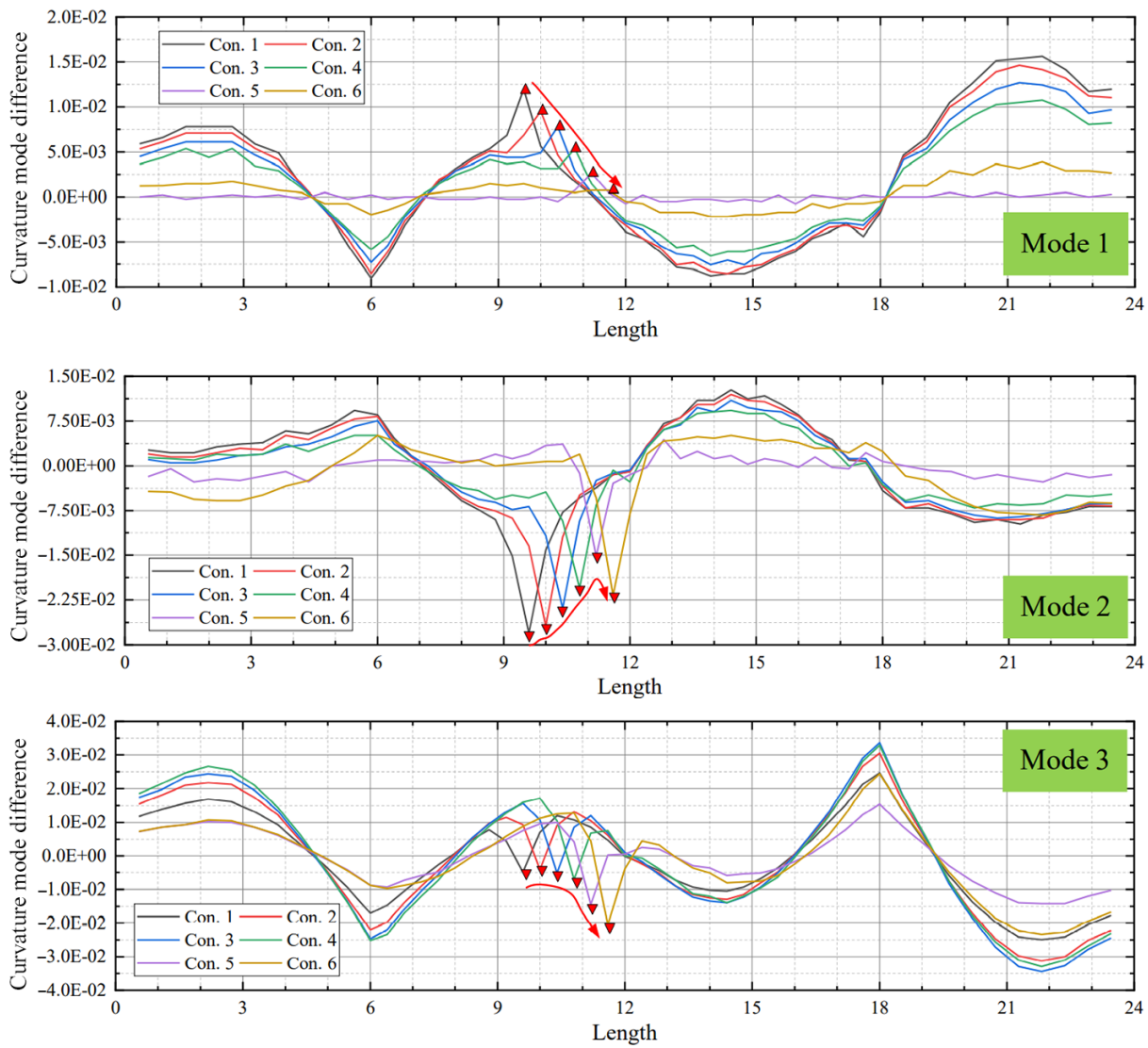

Figure 27. Modal curvature difference of first three order modes under different damage conditions obtained by dense full-field mode shape 
between the fields of view. Through this series of technical means, noncontact measurement and analysis are realized, and the following characteristics are summarized:

(1) Collecting the dynamic information of a bridge structure with a high-speed camera is convenient, fast, and holographic.

(2) (The tracking method proposed in this study does not need to use manual marker for target tracking. The coordinates of each pixel on the lower-edge contour line of the test bridge are extracted from the original frame image and used to constrain the feature point extraction in the machine vision algorithm, then the feature points of the contour line are marked and tracked through this coordinate. Compared with other methods for setting virtual markers, though manual intervention is necessary for the early stage, this method can track the alignment and obtain displacement information of more dense measuring points.

(3) The time history displacement information obtained by the holographic visual sensor and dial gauges is in good agreement and exhibits the same trend, which shows that the holographic visual sensor has sufficient sampling frequency and accuracy to obtain the displacement information of the main beam of the test bridge.

(4) The first three order frequencies of the main beam of the test bridge can be obtained by the holographic visual sensor. Compared with that of the accelerometers, the maximum error of the holographic visual sensor is $2.8 \%$, which fully meets the needs of the engineering project. Although the method proposed in this study is dense measuring points limited by the frame rate of the camera used in the simulation, only the first three order frequencies can be obtained. As the frame rate of the equipment used increases, high frequencies can be obtained.

(5) The first three order modes of the fields of view can be obtained by the technical means adopted in this study, and the mode shape of the entire bridge can be formed by the constraint of the same name pixel point between the fields of view, which is consistent with the measured mode shape trend. However, this method provides more intensive time history information than a traditional contact sensor, and the mode shape is continuous and smooth.

(6) Compared with the traditional method, which requires high-order modal parameters for damage identification, the dense full-field modal parameters obtained by the method proposed in this study can reflect sensitivity to damage location in a low order when used to obtain the modal curvature difference for damage identification. However, damage degree quantification needs further research. The use of modal curvature for damage identification requires high denoising, and the corresponding denoising technology also needs further investigation.

\section{Acknowledgements}

Special thanks to J. L. Heng at the Shenzhen University, and Y. M. Gao at the State Key Laboratory of Mountain Bridge and Tunnel Engineering, and Chongqing Jiaotong University.

\section{Funding}

This research was funded by the foundation as follows:

- This work was supported by the < National Natural Science Foundation of China> under Grant [51778094].

- This work was supported by the < National Science Foundation for Distinguished Young Scholars of China> under Grant [51608080].

- This work was supported by the < National Science Foundation for Distinguished Young Scholars of China> under Grant [51708068].

- This work was supported by the < Chongqing Graduate Scientific Research Innovation Project $>$ under Grant [CYS20287].

\section{Author contributions}

Conceptualization, Z.Z.; formal analysis, G.D.; investigation, X.C.; methodology, G.D.; resources, G.D., S.S. and P.D.; writing - original draft, G.D.; writing - review and editing, G.D. and S.S. All authors have read and agreed to the published version of the manuscript.

\section{Disclosure statement}

The authors declare that we have no competing financial, professional, or personal interests from other parties.

\section{References}

Bao, Y., \& Li, H. (2019). Artificial intelligence for civil engineering. China Civil Engineering, 52(5), 1-11.

Chen, J. G., Davis, A., Wadhwa, N., Durand, F., Freeman, W. T., \& Buyukozturk, O. (2015, September). Video camera-based vibration measurement for condition assessment of civil infrastructure. In NDT-CE International Symposium NonDestructive Testing in Civil Engineering (pp. 15-17), Berlin, Germany.

Chang, C. C., \& Ji, Y. F. (2007). Flexible videogrammetric technique for three-dimensional structural vibration measurement. Journal of Engineering Mechanics, 133(6), 656-664. https://doi.org/10.1061/(ASCE)0733-9399(2007)133:6(656)

Chu, X., Zhou, Z., Deng, G., Duan, X., \& Jiang, X. (2019). An overall deformation monitoring method of structure based on tracking deformation contour. Applied Sciences, 9(21), 4532. https://doi.org/10.3390/app9214532

Deng, G., Zhou, Z., Chu, X., Lei, Y., \& Xiang X. (2018). Method of bridge deflection deformation based on holographic image contour stacking analysis. Science Technology and Engineering, 18(28), 246-253.

Deng, G., Zhou, Z., Shao, S., Chu, X., \& Jian, C. (2020). A novel dense full-field displacement monitoring method based on image sequences and optical flow algorithm. Applied Sciences, 10(6), 2118. https://doi.org/10.3390/app10062118 
Dessi, D., \& Camerlengo, G. (2015). Damage identification techniques via modal curvature analysis: overview and comparison. Mechanical Systems \& Signal Processing, 52-53, 181-205. https://doi.org/10.1016/j.ymssp.2014.05.031

Dong, C. Z., Ye, X. W., \& Jin, T. (2018). Identification of structural dynamic characteristics based on machine vision technology. Measurement, 126, 405-416.

https://doi.org/10.1016/j.measurement.2017.09.043

Dong, C. Z., Celik, O., \& Catbas, F. N. (2019). Marker-free monitoring of the grandstand structures and modal identification using computer vision methods. Structural Health Monitoring, 18(5-6), 1491-1509.

https://doi.org/10.1177/1475921718806895

Eshkevari, S. S., Heydari, N., Kutz, J. N., Pakzad, S. N., Diplas, P., \& Eshkevari, S. S. (2019). Operational vision-based modal identification of structures: A novel framework. In Proceedings of 12th International Workshop on Structural Health Monitoring. SAFRAN. https://doi.org/10.12783/shm2019/32502

Eshkevari, S. S., Pakzad, S. N., Takáč, M., \& Matarazzo, T. J. (2020). Modal identification of bridges using mobile sensors with sparse vibration data. Journal of Engineering Mechanics, 146(4), 04020011.

https://doi.org/10.1061/(ASCE)EM.1943-7889.0001733

Feng, D., Feng, M. Q., Ozer, E., \& Fukuda, Y. (2015). A visionbased sensor for noncontact structural displacement measurement. Sensors, 15(7), 16557-16575. https://doi.org/10.3390/s150716557

Feng, D., \& Feng, M. Q. (2017a). Experimental validation of cost-effective vision-based structural health monitoring. $\mathrm{Me}$ chanical Systems and Signal Processing, 88, 199-211. https://doi.org/10.1016/j.ymssp.2016.11.021

Feng, D., \& Feng, M. Q. (2017b). Identification of structural stiffness and excitation forces in time domain using noncontact vision-based displacement measurement. Journal of Sound and Vibration, 406, 15-28.

https://doi.org/10.1016/j.jsv.2017.06.008

Feng, D., Scarangello, T., Feng, M. Q., \& Ye, Q. (2017). Cable tension force estimate using novel noncontact vision-based sensor. Measurement, 99, 44-52.

https://doi.org/10.1016/j.measurement.2016.12.020

Fleet, D. J., \& Jepson, A. D. (1990). Computation of component image velocity from local phase information. International Journal of Computer Vision, 5(1), 77-104. https://doi.org/10.1007/BF00056772

Goldstein, M. (2003). K_n-nearest neighbor classification. IEEE Transactions on Information Theory, 18(5), 627-630. https://doi.org/10.1109/TIT.1972.1054888

Ho, H. N., Lee, J. H., Park, Y. S., \& Lee, J. J. (2012). A synchronized multipoint vision-based system for displacement measurement of civil infrastructures. The Scientific World Journal, Article ID 519146. https://doi.org/10.1100/2012/519146

Ji, Y. F., \& Chang, C. C. (2008). Nontarget stereo vision technique for spatiotemporal response measurement of line-like structures. Journal of Engineering Mechanics, 134(6), 466-474. https://doi.org/10.1061/(ASCE)0733-9399(2008)134:6(466)

Jiang T., Frøseth G. T., Rønnquist A., \& Fagerholt, E. (2020). A robust line-tracking photogrammetry method for uplift measurements of railway catenary systems in noisy backgrounds. Mechanical Systems and Signal Processing, 144, 106888. https://doi.org/10.1016/j.ymssp.2020.106888

Li, H., Huang, Y., Ou, J., \& Bao, Y. (2011). Fractal dimensionbased damage detection method for beams with a uniform cross-section. Computer-Aided Civil and Infrastructure Engineering, 26(3), 190-206.

https://doi.org/10.1111/j.1467-8667.2010.00686.x
Malekjafarian, A., McGetrick, P. J., \& OBrien, E. J. (2015). A review of indirect bridge monitoring using passing vehicles. Shock and Vibration, Article ID 286139.

https://doi.org/10.1155/2015/286139

Martins, L. L., Rebordão, J. M., \& Ribeiro, A. S. (2013). Conception and development of an optical methodology applied to long-distance measurement of suspension bridges dynamic displacement. Journal of Physics: Conference Series, 459(1), 012055. https://doi.org/10.1088/1742-6596/459/1/012055

Matarazzo, T. J., \& Pakzad, S. N. (2018). Scalable structural modal identification using dynamic sensor network data with STRIDEX. Computer-Aided Civil and Infrastructure Engineering, 33(1), 4-20. https://doi.org/10.1111/mice.12298

Matas, J., \& Chum, O. (2004). Randomized RANSAC with $T_{d, d}$ test. Image and Vision Computing, 22(10), 837-842. https://doi.org/10.1016/j.imavis.2004.02.009

Mezirow, J. (2014). Perspective transformation. Adult Education, 28(2), 100-110. https://doi.org/10.1177/074171367802800202

Sarrafi, A., Poozesh, P., Niezrecki, C., \& Mao, Z. (2017, April). Mode extraction on wind turbine blades via phase-based video motion estimation. In Smart Materials and Nondestructive Evaluation for Energy Systems 2017 (Vol. 10171). International Society for Optics and Photonics. https://doi.org/10.1117/12.2260406

Shao, S., Zhou, Z., Deng, G., Du, P., Jian, C., \& Yu, Z. (2020). Experiment of structural geometric morphology monitoring for bridges using holographic visual sensor. Sensors, 20(4), 1187. https://doi.org/10.3390/s20041187

Wadhwa, N., Rubinstein, M., Durand, F., \& Freeman, W. T. (2013). Phase-based video motion processing. ACM Transactions on Graphics (TOG), 32(4).

https://doi.org/10.1145/2461912.2461966

Wang, S., Zhou, Z., Wen, D., \& Huang, Y. (2016). New method for calculating the preoffsetting value of the saddle on suspension bridges considering the influence of more parameters. Journal of Bridge Engineering, 21(12), 06016010. https://doi.org/10.1061/(ASCE)BE.1943-5592.0000956

Xu, Y., \& Brownjohn, J. M. (2018). Review of machine-vision based methodologies for displacement measurement in civil structures. Journal of Civil Structural Health Monitoring, 8(1), 91-110. https://doi.org/10.1007/s13349-017-0261-4

Yang, Y. B., Lin, C. W., \& Yau, J. D. (2004). Extracting bridge frequencies from the dynamic response of a passing vehicle. Journal of Sound and Vibration, 272(3-5), 471-493. https://doi.org/10.1016/S0022-460X(03)00378-X

Yang, Y., Dorn, C., Mancini, T., Talken, Z., Kenyon, G., Farrar, C., \& Mascareñas, D. (2017a). Blind identification of full-field vibration modes from video measurements with phase-based video motion magnification. Mechanical Systems and Signal Processing, 85, 567-590.

https://doi.org/10.1016/j.ymssp.2016.08.041

Yang, Y., Dorn, C., Mancini, T., Talken, Z., Nagarajaiah, S., Kenyon, G., Farrar, C., \& Mascareñas, D. (2017b). Blind identification of full-field vibration modes of output-only structures from uniformly-sampled, possibly temporally-aliased (subNyquist), video measurements. Journal of Sound and Vibration, 390, 232-256. https://doi.org/10.1016/j.jsv.2016.11.034

Yang, Y., Dorn, C., Mancini, T., Talken, Z., Theiler, J., Kenyon, G., Farrar, C., \& Mascarenas, D. (2018a). Reference-free detection of minute, non-visible, damage using full-field, highresolution mode shapes output-only identified from digital videos of structures. Structural Health Monitoring, 17(3), 514-531. https://doi.org/10.1177/1475921717704385 
Yang, Y., Dorn, C., Mancini, T., Talken, Z., Kenyon, G., Farrar, C., \& Mascareñas, D. (2018b). Spatiotemporal videodomain high-fidelity simulation and realistic visualization of full-field dynamic responses of structures by a combination of high-spatial-resolution modal model and video motion manipulations. Structural Control and Health Monitoring, 25(8), e2193. https://doi.org/10.1002/stc.2193

Ye, X. W., Dong, C. Z., \& Liu, T. (2016a). Image-based structural dynamic displacement measurement using different multiobject tracking algorithms. Smart Structures and Systems, 17(6), 935-956. https://doi.org/10.12989/sss.2016.17.6.935

Ye, X. W., Dong, C. Z., \& Liu, T. (2016b). A review of machine vision-based structural health monitoring: methodologies and applications. Journal of Sensors, Article ID 7103039. https://doi.org/10.1155/2016/7103039

Ye, X. W., Ni, Y. Q., Wai, T. T., Wong, K. Y., Zhang, X. M., \& $\mathrm{Xu}, \mathrm{F}$. (2013). A vision-based system for dynamic displacement measurement of long-span bridges: algorithm and verification. Smart Structures and Systems, 12(3-4), 363-379. https://doi.org/10.12989/sss.2013.12.3_4.363

Ye, X. W., \& Dong, C. Z. (2019). Review of computer visionbased structural displacement monitoring. China Journal of Highway and Transport, 32(11), 21-39.

Zhang, Z. (2000). A flexible new technique for camera calibration. IEEE Transactions on Pattern Analysis and Machine Intelligence, 22(11), 1330-1334. https://doi.org/10.1109/34.888718

Zhang, Z. (2004). Camera calibration with one-dimensional objects. IEEE Transactions on Pattern Analysis and Machine Intelligence, 26(7), 892-899. https://doi.org/10.1109/TPAMI.2004.21

Zhao, J., Bao, Y., Guan, Z., Zuo, W., Li, J., \& Li, H. (2019). Videobased multiscale identification approach for tower vibration of a cable-stayed bridge model under earthquake ground motions. Structural Control and Health Monitoring, 26(3), e2314. https://doi.org/10.1002/stc.2314 